\title{
Catch the next designer drug: bioactivity data derived pharmacological affinity fingerprints and performance in similarity searching and unsupervised clustering
}

Kedan He ( $\square$ nina.kh23@gmail.com )

Eastern Connecticut State University https://orcid.org/0000-0002-1953-4956

\section{Research Article}

Keywords: New psychoactive substances, Pharmacological affinity fingerprint, Bioactivity data, Similarity search, Unsupervised clustering, Machine learning

Posted Date: February 22nd, 2022

DOI: https://doi.org/10.21203/rs.3.rs-1316376/v1

License: (9) This work is licensed under a Creative Commons Attribution 4.0 International License. Read Full License 


\title{
Title: Catch the next designer drug: bioactivity data derived
}

\section{pharmacological affinity fingerprints and performance in similarity searching and unsupervised clustering}

\author{
Kedan $\mathrm{He}^{*}$ \\ *Eastern Connecticut State University, Physical Sciences, 83 Windham St, Willimantic, CT 06226, \\ USA. Email: hek@easternct.edu. Phone: 860-465-5759
}

\begin{abstract}
Facing the continuous emergence of new psychoactive substances (NPS) and their threat to public health, more effective methods for NPS prediction and identification are critical.

In this study, the pharmacological affinity fingerprints $(P h-f p)$ of NPS compounds were predicted by Random Forest classification models using bioactivity data from the ChEMBL database. The binary $\mathrm{Ph}-\mathrm{f}$ is the vector consisting of a compound's activity against a list of molecular targets reported to be responsible for the pharmacological effects of NPS. Their performance in similarity searching and unsupervised clustering was assessed and compared to 2D structure fingerprints Morgan and MACCS (1024-bits ECFP4 and 166-bits SMARTS-based MACCS implementation of RDKit). The performance in retrieving compounds according to their pharmacological categorizations is influenced by the predicted active assay counts in $P h-f p$ and the choice of similarity metric. Overall, the comparative unsupervised clustering analysis shows that $\mathrm{Ph}-\mathrm{p}$ constructed using classification models using Morgan fingerprints gives satisfactory performance comparable to Morgan according to external and internal clustering validation indices.
\end{abstract}

Keywords: New psychoactive substances, Pharmacological affinity fingerprint, Bioactivity data, Similarity search, Unsupervised clustering, Machine learning 


\section{Introduction}

"Designer drugs" or new psychoactive substances (NPS) are compounds that slightly modify the molecular structure of existing controlled substances to mimic their pharmacological effects and bypass legislation. ${ }^{[1,2]}$ Terms such as "research chemicals, bath salts, fertilizers, incense, and plant foods" are used to circumvent legislation designed to control the supply and distribution of these substances. According to the United Nations Office on Drugs and Crime (UNODC), 126 countries have reported a total of more than 1,047 NPS as of December $2020 .{ }^{[1]}$ Using a $24 / 7$ web crawler to capture the real number of NPS shows over 4,000 unique substances of interest circulating in the online environment, a number roughly four times greater than that reported in known NPS databases. ${ }^{[3]}$ Many countries have used or amended existing legislation, or innovative legal instruments, as a way to address the prevalence of NPS. For example, the Controlled Substances Act, passed in 1986 in the United States, allows any chemical that is "substantially similar" to a Schedule I or II controlled substance to be treated as a Schedule I substance. ${ }^{[2]}$ In the UK, any substance that is not regulated by the Misuse of Drugs Act 1971 falls within the scope of the Psychoactive Substances Act 2016. ${ }^{[4]}$ However, the ban on any particular NPS or NPS category has led to a rapid substitution in the market. Given that these compounds will now reach users through more clandestine routes and that synthetic drug overdose mortality is increasing across all age groups, races, genders, and ethnicities, new tools and methods must be developed to more effectively address the current problem of NPS abuse. ${ }^{[5]}$

NPS are a heterogeneous group of substances, often classified according to their chemical scaffoldings, based on the observation that structurally similar compounds may have similar biological activities and exhibit similar spectral behavior. ${ }^{[6]}$ Systematic classification of NPS based on pharmacological effects is very challenging because a drug often interacts with many different biological targets. There is no universally agreed method for classifying NPS, and based on their 
primary mechanism of action and molecular targets, they have generally been grouped into four somewhat overlapping functional categories related to their chemical structure and pharmacological effects: stimulants, cannabinoids, hallucinogens, and depressants. ${ }^{[7-9]}$ It is very likely that some new compounds do not neatly fit into these 'four-category' classification and their effects cross these boundaries. The currently used 'four-category' classification system groups together compounds with highly varied chemical structures (such as the synthetic cannabinoids), or mechanistically heterogeneous compounds (such as the depressants) in a practical workable system for clinicians, scientists, law enforcement agencies and other interested parties.

Synthetic stimulants currently represent the largest group of NPS that are monitored by the UNODC and EMCDDA. ${ }^{[1,10]}$ It include cathinones, aminoindanes, benzofurans, phenethylamines, piperazines, and tryptamines, of which synthetic cathinones are by far the largest group and the most studied. Synthetic stimulants exert their stimulatory effects by increasing the concentrations of the monoamine neurotransmitters dopamine (DA), serotonin (5HT), and to a lesser extent, norepinephrine (NE) in the synaptic cleft. ${ }^{[1,12]}$ There are two distinct mechanisms of synthetic stimulants: stimulation of neurotransmitter release from the cytosolic pool or synaptic vesicles through inhibition of vesicular monoamine transporter- 2 and reversal of transporter influx; ${ }^{[13]}$ and inhibition of neurotransmitters uptake from the synaptic cleft through inhibition of the plasma membrane transporters. ${ }^{[14-16]}$ Synthetic cannabinoids were first formally identified and reported to EMCDDA in 2008. Synthetic cannabinoids represent the largest and most structurally diverse class of designer drugs, and some of these compounds are similar to phyto- and endocannabinoids. Synthetic cannabinoids interact primarily with the endocannabinoids systems and its G-proteincoupled cannabinoid receptor type-1 (CB1) and occasionally cannabinoid receptor type-2 (CB2). ${ }^{[17,18]}$ The current hypotheses on how synthetic cannabinoids modulate their effects via these receptors and the difference between the observed clinical effects of traditional cannabis and synthetic 
cannabinoids include biased signaling at cannabinoid receptors or the disruption of mitochondrial homeostasis. ${ }^{[19,20]}$ Synthetic hallucinogens from the phenethylamine and tryptamine classes, also known as serotonergic psychedelics, interact primarily with cortical serotonin receptors can inhibit the reuptake and increase the release of serotonin, but display heterogeneous profile at several receptors. ${ }^{[21-23]}$ The 5-HT2A receptor agonism plays a key role in mediating the psychedelic effects of both phenethylamine and tryptamine compounds, ${ }^{[24]}$ but the concurrent activation of 5-HT1A receptors has been suggested to contribute to the qualitative effects of tryptamine psychedelics and distinguishing them from phenethylamine psychedelics. ${ }^{[25]}$ Affinity for 5-HT2A and 5-HT2C receptors is also reported correlated with the dose that induces psychedelic effects in humans. ${ }^{[26]}$ Most synthetic hallucinogens have been shown to interact with other monoaminergic targets, including adrenergic, dopaminergic, and histaminergic receptors. ${ }^{[21,22,27-29]}$ Unlike phenethylamine, many tryptamines interact with monoamine transporters at pharmacologically relevant concentrations. ${ }^{[28,30,31]}$ Synthetic depressants are broadly classified into two sub-categories: synthetic benzodiazepines and synthetic opioids. Synthetic benzodiazepines mediate their effects through interactions at gamma-aminobutyric acid-A (GABA-A) receptors, ion channels that consist of different subunit compositions, responding to the inhibitory neurotransmitter GABA. ${ }^{[32,33]}$ Synthetic opioids are created to bind to the same receptors in the brain as opiates, such as morphine and codeine, and produce similar effects such as euphoria, anxiolysis, feelings of relaxation and drowsiness. ${ }^{[34]}$ Novel fentanyl analogs and other synthetic opioids interact with $G$ protein-coupled opioid receptors as partial to full agonists at $\mu$-, $\delta$-, and $\kappa$-opioid receptor subtypes, with selectivity for the $\mu$-opioid receptor. ${ }^{[35-37]}$

According to the recommendations of the Advisory Council on the Misuse of Drugs (ACMD), the in vitro testing should be used to demonstrate whether a substance is psychoactive. ${ }^{[38]}$ The use of structural similarities to identify compounds with similar biological activities has been the 
subject of virtual screening (VS) strategies. ${ }^{[39]}$ Two-dimensional (2D) molecular structure fingerprints have been successfully combined with statistical and machine learning methods for predicting target binding and other properties of molecules. ${ }^{[40]}$ However, ligand-based similarity search methods perform poorly when the number of known ligands is insufficient, such as when there are far more unknown NPS compounds than known NPS compounds. For example, synthetic cannabinoids interact less ambiguously with CB1 receptors but containing very structurally diverse molecules. Synthetic cannabinoids demonstrate limited structural similarity to d9-THC are referred to as synthetic cannabinoids due to their pharmacological mechanisms. ${ }^{[41]}$ Therefore, unless specifically included in reference databases they will typically not be detected in conventional drug screening procedures such as urine tests. ${ }^{[42]}$ Activity cliff, on the other hand, is generally defined as a pair of structurally similar compounds with a large difference in potency. 5F-PY-PICA (PubChem CID 129520948) and 5F-PY-PINACA (PubChem CID 125181281) were identified in 2015 and regarded as putative synthetic cannabinoid receptor agonist. However, both compounds exhibited low affinity and efficacy at $\mathrm{CB} 1$ and $\mathrm{CB} 2$ receptors in vitro, and failed to elicit the in vivo effects potently induced by other synthetic cannabinoids, which cast doubt on their classification as synthetic cannabinoid receptor agonists. ${ }^{[43]}$ Because of the scarcity of studies on the interaction of synthetic cannabinoids with non-cannabinoid targets, potential effects on non-cannabinoid receptors and different signaling pathways that have yet to be identified cannot be ruled out. ${ }^{[19,44]}$

In contrast to molecular fingerprint, where it reflects compounds' chemical structure, the socalled bioactivity profile can be used to quantitatively describe compound interactions with the proteome without taking its chemical structure into account. ${ }^{[45]}$ It was demonstrated for compounds that interact with multiple targets that the comparison by their bioactivity profile rather than by their structures can lead to discovery structurally dissimilar compounds eliciting same biological responses. ${ }^{[46]}$ Several studies have reported that using publicly available bioactivity data to construct 
such bioactivity fingerprints performs better and has a higher hit rate in classification tasks compared to ECFP4 fingerprints. ${ }^{[47-49]}$ HTSFPs report the activities of small molecules in 243 different PubChem bioassays and are used to retrieve hits that are structurally diverse and different from the active compounds retrieved by chemical similarity-based methods. ${ }^{[49]}$

This study aims to investigate the potential of using fingerprints that encode the compound's bioactive profiles when applied to unsupervised classification methods, also known as clustering, for the selection of representative compounds. Given a set of data points $X_{i}, \ldots, X_{n}$ and some notion of similarity $s_{i j}>0$ between all pairs of data points $X_{i}, X_{j}$, the intuitive goal of clustering is to divide the data points into several groups (clusters) such that points in the same group are similar and points in different groups are dissimilar to each other. One of the main limitations of the widely used $k$-Means is the need for a priori setting of the number of clusters $(K)$. This method is also not recommended in cases where the size of the clusters is very different. On the other hands, the hierarchical clustering take into account the linkage between data points called a dendrogram, which represents an ensemble of clustering models with every possible $K$. Hierarchical clustering approaches require defining a dissimilarity function and a linkage criterion. Agglomerative clustering is initialized by considering every object as a different cluster to create $N$ singleton clusters. Then the closest two objects are combined, leaving $N-1$ clusters. In each step of the algorithm, which pair of clusters is linked is determined by the linkage criteria, which will greatly affect the results. The dendrogram comprising the clustering model can then be "cut" for any number of clusters $2 \leq K \leq$ $N$. Recently, spectral clustering has attracted great interest in the analysis of biological and chemical data. ${ }^{[50-53]}$ If we do not have more information than similarities between data points, a nice way of representing the data is in form of the similarity graph $G=(V, E)$. Each vertex $v_{i}$ in this graph represents a data point $X_{i}$. Two vertices are connected if the similarity $s_{i j}$ between the 
corresponding data points $X_{i}$ and $X_{j}$ is positive or larger than a certain threshold, and the edge is weighted by $s_{i j}$. The problem of clustering can now to reformulated using the similarity graph: to find a partition of the graph such that the edges between different groups have very low weights (which means that points in different clusters are dissimilar from each other) and the edges within a group have high weights (which means that points within the same cluster are similar to each other). When constructing similarity graphs the goal is to model the local neighborhood relationships between the data points. A reasonable default candidate of the similarity function is the Gaussian similarity function $s_{i j}=\exp \left(-\frac{\left\|d_{i j}\right\|^{2}}{2 \sigma^{2}}\right)$ with the Euclidean distance $d\left(X_{i}, X_{j}\right)$. The graph Laplacian matrix is defined as the difference of two matrices as $L=D-W$, where $D$ is the diagonal degree matrix and $W$ is a matrix of positive weights assigned to the graph edges. The eigenvectors of the normalized graph Laplacian then are used as input for a $k$-Means clustering step for final partition. ${ }^{[54]}$

To take advantage of a large amount of bioactivity data in the ChEMBL database, a pharmacological affinity fingerprint $(P h-f p)$ was developed based on Random Forest (RF) classification models. The RF classification model was trained and cross-validated using bioassay data covering a range of molecular targets that are informative for the pharmacological characterization of the NPS. The $P h-f p$ is much shorter in terms of bit-length compared to conventional molecular structural fingerprints. The similarity of the pharmacological profile of compounds can be quantified by a metric similar to the commonly used Tanimoto coefficient. Since $P h-f p$ is defined in a data-driven manner, it can be updated and adapted to the continuous availability of bioassay data in the public domain. An external NPS set was used to compare the performance of Ph-fp with Morgan and MACCS fingerprints in two tasks: similarity search and unsupervised clustering. Both clustering algorithms were parameterized and evaluated using internal and external 
indices. In particular, we have been interested in addressing the question to what extent the datadriven $P h-f p$ can indeed be used to identify compounds based on classical pharmacological categorization, rather than on their biological activity against a single target.

\section{Methods and Materials}

\section{Dataset acquisition and curation}

The ChEMBL database, version 29, was used as the data source. ${ }^{[55,56]}$ A range of major neurotransmitter receptors and transporters were selected for the in vivo pharmacological characterization of NPS compounds..$^{[9,16,24,27,43,57-60]}$ The biological activity of a compound is quantified by its affinity (given as Ki) and/or its potency (given as IC50/EC50). Bioactivity data for both human and non-human targets were considered. Each distinct molecular target is defined by its unique UniProtKB ID, and each organism/target/activity type combination is referred to as an assay and separate models were built for each assay dataset. ChEMBL bioactivity data were filtered using the following criteria: (1) only single protein target type is considered; (2) human and nonhuman organisms [Homo sapiens, Rattus norvegicus, Mus musculus] are considered; (3) activity types of only Ki, IC50, or EC50; (4) assay type is "Binding"; (5) activity relationship defined as "="; (6) activity values reported in standard units nM; (7) MW up to 900. The mean standard activity values were calculated when multiple activity records are available. Assays with less than 50 distinct compounds were discarded. Active compounds were defined as those with $\mathrm{pK} i, \mathrm{p} I C 50$, or pEC50 better than or equal to an affinity cutoff value. For each active compound, 4 decoys were randomly sampled from the benchmarking DUD-E (DUD-Enhanced) database ${ }^{[61]}$ to ensure that the dataset for each assay was reasonably sized and suitable for comparing the performance of machine learning classification models, while avoiding the creation of highly unbalanced data sets. The DUD-E ${ }^{[61]}$ 
decoy compounds were extracted from the ZINC database ${ }^{[62]}$ and filtered based on physicochemical properties. A topological dissimilarity filter was also applied to avoid active compounds in the decoy sets. As an additional step randomly sampled decoys with Tanimoto similarity coefficient larger than 0.9 were removed. The list of assays used to train each model is available from GitHub repository.

The Molecular ACCess System (MACCS) and Morgan fingerprints as implemented in the RDKit toolkit were calculated as the molecular descriptors and used as input feature for the classification model. The substructural key-based fingerprints, MACCS, encodes the absence (0) and presence (1) of predefined chemical features, and is represented by a 166 binary bitstring. ${ }^{[63]}$ MACCS have been shown to be more discriminating than structural key fingerprints using many more features. ${ }^{[63,64]}$ Morgan is the RDKit implementation of the ECFP4 extended connectivity fingerprint with radius 2 as 1024-bit vector. ${ }^{[65]}$ Extended connectivity fingerprints haven shown the best performance in comparative tests including virtual screening, ${ }^{[66]}$ scaffold-hopping, ${ }^{[67]}$ and clustering $^{[68]}$.

\section{Model training, validation, and performance evaluation}

Random Forest $(\mathrm{RF})^{[69,70]}$ classification model was constructed using the ensemble.RandomForestClassifier module from the Python scikit-learn library. The number of decision trees used was set to $[20,60,100,140,180]$ and the maximum number of features as the total number of features. Ten-fold Nested cross-validation (CV) is used in model training and validation. Each assay dataset was split into training and test sets with 90:10 ratio using the model_selection.KFold module of scikit-learn. The training set was used for hyperparameter tuning and then the model was validated with the test set. This process was repeated 10 times by selecting a different $10 \%$ of the data for validation and by using a different $90 \%$ of the data to develop a new model from scratch. The overall performance was then calculated as a mean of classification performances of the 10 separately developed models on different $10 \%$ sets of the validation data. The nested CV ensure that 
the data used to validate the classifier is not part of the data used to train it, which provides almost unbiased performance estimates. ${ }^{[11]}$

The Matthews correlation coefficient (MCC) was utilized to measure and compare the performance of classification models trained in this study: $:^{[2]}$

$$
M C C=\frac{\mathrm{tp} \cdot \mathrm{tn}-\mathrm{fp} \cdot \mathrm{fn}}{\sqrt{(\mathrm{tp}+\mathrm{fp})(\mathrm{tp}+\mathrm{fn})(\mathrm{tn}+\mathrm{fp})(\mathrm{tn}+\mathrm{fn})}}
$$

where tp: true positive, tn: true negative, fp: false positive (Type I error), fn: false negative (Type II error). MCC incorporates the imbalance of the dataset and its invariance to the exchange of classes and is therefore considered a balanced measure of the biased data set. ${ }^{[73]}$ Independent of their ratio in the dataset, the classifier must make correct predictions for both negative and positive cases to obtain a high MCC. It ranges in the interval of $[-1,+1]$ and reaches the extreme values of -1 and +1 in the case of complete misclassification and perfect classification, respectively, while MCC $=0$ is the expected value of the coin tossing classifier.

\section{Pharmacological affinity fingerprint $(P h-f p)$ construction}

The $P h-\not p$ of a compound is a binary array containing the compound's activity across the list of target assays predicted by corresponding classification models. Only models with MCC $\geq 0.90$ were included in the construction of the $\mathrm{Ph}-\mathrm{f}$. For each assay, the predictions were repeated 50 times using randomly sampled $90 \%$ of the ChEMBL data as training sets. The final prediction is aggregated by majority voting using sklearn.ensemble.BaggingClassifier. The workflow for the construction of the $P h-f p$ is shown in Fig 1.

The NPS set includes 189 compounds collected from the literature and their pharmacological classification was determined based on their in vitro profile data. ${ }^{[22,27,28,57-60,74-77]}$ Twenty-one natural and synthetic alkaloid and phenylpiperidine opioids and 13 benzodiazepines are classified as depressants; 33 cathinones, 16 phenethylamines, 10 benzofurans, 9 piperidines, 5 
aminoindanes are classified as stimulants; 8 THC and derivatives, 14 indoles, and 7 indazole are classified as cannabinoids; 39 phenethylamines (ring-substituted phenethylamines including 2C drugs and their methoxybenzyl [NBOMes] analogues) and 14 tryptamine are classified as serotonergic psychedelics. In this study, the two sub-groups of depressants were separated as individual class because of the unique molecular targets reported in pharmacological studies.

Table 1 NPS set compounds pharmacological categorization and primary molecular target

\begin{tabular}{|c|c|c|}
\hline Pharmacological Category $^{[7,12]}$ & Target(s) & Actives \\
\hline Stimulants & NET, DAT, SERT & 73 \\
\hline Cannabinoids & CB1, CB2 & 29 \\
\hline Serotonergic psychedelics & 5-HT $_{2 \mathbf{A}}, \mathbf{5 - H T _ { 2 C }}$ & 53 \\
\hline Depressant - opioids & $\boldsymbol{\mu}$-opioid & 21 \\
\hline Depressant - benzodiazepines & GABA $_{\mathbf{A}}$ & 13 \\
\hline
\end{tabular}




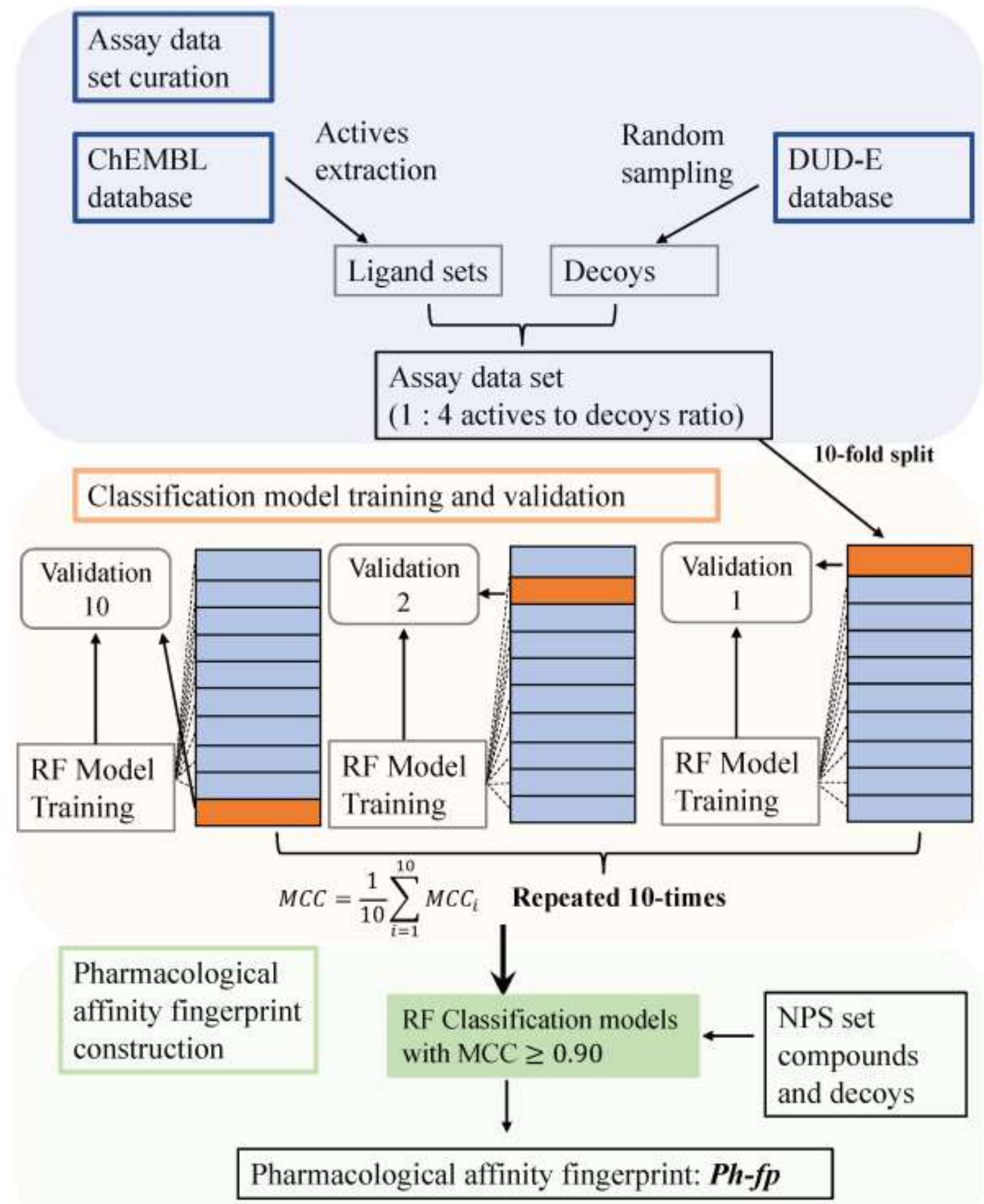

Fig. 1 The workflow for the construction of binary pharmacological affinity fingerprint A total of 132 assay datasets for 70 unique molecular targets were extracted from the ChEMBL 29 database ${ }^{[55,56]}$ Each RF classification model was trained with $90 \%$ of the data, validated by $10 \%$ of the data, and repeated 10 times (10-fold Nested CV). Only models with a mean MCC greater than or equal to 0.90 were included in the $P h-f p$ construction for the NPS set compounds. 


\section{Pharmacological affinity fingerprint in similarity search performance assessment}

The similarity of the pharmacological profiles of the molecules described by $\mathrm{Ph}-\mathrm{fp}$ was calculated by the Rogot-Goldberg index: ${ }^{[78]}$

$$
S_{R G}=\frac{a}{2 a+b+c}+\frac{d}{2 d+b+c}
$$

The four basic quantities can be calculated for each pair of fingerprints are:

$a$ : the number of 1's (common “on" bits).

$b$ : the number of 1's present in the first fingerprint but absent in the second

$c$ : the number of 1's present in the second fingerprint but absent in the first

$d$ : the number of coincident 0 's (common “off” bits)

In comparison to the frequently used Tanimoto coefficient ${ }^{[79]}$ for structural similarity

calculated using binary fingerprint, the Rogot-Goldberg index values the information at which targets the compound is inactive as well as which at targets it is active.

The similarity search performance was assessed by two quality metrics, AUC and EF10.

AUC is the area under the ROC curve and it quantifies the general ability of a method to discriminate between actives and inactives. AUC equals to the probability that a classifier will rank a randomly chosen positive instance higher than a randomly chosen negative example. Enrichment factor, EF, explicitly measures the early recognition performance. EF is defined as:

$$
E F(\chi \%)=\frac{\frac{P_{\chi \%}}{N_{\chi \%}}}{\frac{P_{\text {total }}}{N_{\text {total }}}}
$$

where $\chi \%$ is the fraction of the sorted dataset EF is calculated for, $P_{\chi \%}$ is the number of actives in this fraction and $N_{\chi \%}$ is the number of all molecules in this fraction, whereas $P_{\text {total }}$ and $N_{\text {total }}$ are 
the number of actives and the total number of molecules in the dataset. In this study, EF10 at top $10 \%(\chi=0.10)$ of the sorted data set was calculated.

The performance of the $\mathrm{Ph}-\mathrm{f}$ in similarity searching was evaluated using the NPS set and compared with Morgan and MACCS fingerprints. For each experiment, 50 similarity searches were performed for each pharmacological class using different randomly selected test sets where actives were defined as NPS compounds from the pharmacological class of interest. The query set consisted of 10 actives, and 10 decoys randomly selected for each active. The remaining actives of this class and 10 randomly sampled decoys for each active formed the test set to maintain the same 10:1 decoy

to actives ratio. The fraction of actives in the test set $\left(\frac{P_{\text {total }}}{N_{\text {total }}}\right)$ is kept constant at 0.091 . For each compound in the test set, its similarity to the query compound is calculated, and its nearest neighbor with the highest similarity is retained. The entire test set is then sorted by decreasing similarity and the AUC and EF10 are calculated based on this sorted list.

\section{Pharmacological affinity fingerprint in clustering performance assessment}

The NPS set was transformed into an $n \times n$ matrices $(n=189)$ using structural similarity calculated using MACCS or Morgan structural fingerprints or pharmacological similarity calculated using $P h-f p$ and submitted to hierarchical and spectral clustering algorithms. In agglomerative hierarchical clustering, four linkage criteria are tested: Ward, complete, weighted average, and single linkage, which measure the proximity between two clusters. In spectral clustering, the $\mathrm{n} \times \mathrm{n}$ similarity matrix is transformed into a similarity graph in the form of an affinity matrix which is represented by $A$ in different manners: 1$). k$-nearest neighbor graph by connect each point with $k$-nearest neighbors. After connecting the appropriate vertices, the edges are weighted by the similarity of their endpoints. 2). Fully connected graph simply connects all points with positive similarity with each other and weight all edges by $s_{i j}$. Compute the first $K$ generalized eigenvectors $u_{1}, \ldots, u_{K}$ of the 
generalized eigenproblem $L u=\lambda D u$, where the generalized eigenvectors of $L$ correspond to the eigenvectors of the matrix $L_{r w}=D^{-1} L=I-D^{-1} W$. These eigenvectors are used as input in the last $k$-Means step to extract the final partition. The main trick is to change the representation of the abstract data points $X_{i}$ to points $y_{i} \in \mathbb{R}^{K}$. The clustering hyperparameters investigated are listed in Table 2.

Table 2 Clustering hyperparameters investigated

\begin{tabular}{|c|c|c|}
\hline Hyperparameters & Parameter & Values explored \\
\hline \multicolumn{3}{|l|}{ Hierarchical clustering } \\
\hline \multirow[t]{4}{*}{ Linkage } & Ward & Minimizes the variance of the clusters being merged \\
\hline & Complete & Maximum distances between all observations of the two sets \\
\hline & Average & Average of the distances of each observation of the two sets \\
\hline & Single & Minimum distances between all observations of the two sets \\
\hline \multicolumn{3}{|l|}{ Spectral clustering } \\
\hline \multirow{2}{*}{$\begin{array}{l}\text { Fully connected } \\
\text { graph }(\mathrm{RBF})\end{array}$} & $\gamma$ & {$[1,2,3,4,5]$} \\
\hline & eigen_tol & {$[0.1,0.01,0.001,0.0001,0.00001,0.000001]$} \\
\hline \multirow{2}{*}{$\begin{array}{l}k \text {-nearest neighbor } \\
\text { graph }\end{array}$} & n_neighbors & {$[7,9,11,13,15,17,19]$} \\
\hline & eigen_tol & {$[0.1,0.01,0.001,0.0001,0.00001,0.000001]$} \\
\hline
\end{tabular}

The fcluster and dendrogram in scipy.cluster.bierarchy package are used for hierarchical clustering, the SpectralClustering in sklearn.cluster package are used for spectral clusterings

The Leave-one-out cross validation was used in all the clustering analysis and the averaged results of n iterations were reported. Both internal and external indices were used to measure the quality of the clustering partition. The internal indices Silhouette score ${ }^{[80]}$ estimate the quality of a partition by measuring how closely each instance is related to the cluster and how well-separated a cluster is from other clusters given the number of desired clusters $K$. Silhouette score ranges from -1 to +1 , where +1 means clusters are well apart from each other and clearly distinguished, -1 indicates member is assigned to the wrong cluster. On the other hand, external validation indices measure the similarity between the output of the clustering algorithm and the correct partitioning of the dataset. ${ }^{[81]}$ In this study the clustering success defined as correctly identify the Maximum Common Substructure (MCS) based clusters and/or the five pharmacological classes are evaluated 
using the adjusted Rand-Index (ARI) ${ }^{[82]}$ and the normalized mutual information (NMI) ${ }^{[83]}$. When two sets of cluster labels have a perfect one-to-one correspondence, the ARI equal to unity. NMI $=0$ mean two partitions contain no information about one another, whereases NMI $=1$ indicates two partitions contain perfect information about one another. See Supporting document for more detail.

All hierarchical clusterings were generated using the fcluster and dendrogram in scipy.cluster.hierarchy package; spectral clusterings were conducted using the SpectralClustering in sklearn.cluster package; Silhouette score, ARI, and NMI values were computed using sklearn.metrics package.

\section{Results and Discussion}

\section{Data curation and statistics}

A total of 132 data sets were curated using ChEMBL and DUD-E databases, covering 70 distinct molecular targets from 11 classes (see Supporting Document on GitHub repository), among which 48 targets were modeled with more than one assay. Three different affinity measurements (pKI, pIC50 and pEC50) with cutoff values greater than or equal to $5(10 \mu \mathrm{M}), 6(1 \mu \mathrm{M})$ and $7(10$ $\mathrm{nM}$ ) were used to define the active compound. When a tighter affinity cutoff was applied, fewer assay datasets were used during model training due to fewer compounds labeled as active. Assay datasets with less than 50 unique active compounds were further discarded, resulting in 132, 126, and 116 models being built when using cutoff 5, 6, and 7, respectively. To construct the binary $P h-f p$ for the NPS set compounds, only the models with MCC $\geq 0.90$ were included to ensure sufficiently high predictive power. Six versions of the $P h-f p$ were constructed using assay datasets created with different affinity cutoff values and molecules encoded by two molecular descriptors, which are referred in the following text as $p 5 \_$maccs, $p 6 \_$maccs, $p 7 \_$maccs, $p 5 \_$morgan, $p 6 \_$morgan, and $p 7 \_m o r g a n$, their final lengths are listed in Table 3. The comparison of the molecular target classes between the 
total assay datasets trained and final models included in each $P h-f p$ is shown in Fig 2 using activity cutoff values greater than or equal to 5 , which shows that the distribution are preserved in the final $P h-p$.

Table 3 Number of assay datasets used in the RF classification model and final length of Ph-fp

\begin{tabular}{|c|c|c|c|}
\hline & $\mathbf{5 ( 1 0} \boldsymbol{\mu M})$ & $\mathbf{6}(\mathbf{1} \boldsymbol{\mu M})$ & $\mathbf{7}(\mathbf{1 0} \mathbf{n M})$ \\
\hline Total assay sets & 132 & 126 & 116 \\
\hline \multicolumn{4}{|c|}{ Final $\boldsymbol{P h}$-fp using different molecular descriptors } \\
\hline MACCS (116 bits) & 113 & 110 & 102 \\
\hline Morgan (1024 bits) & 107 & 106 & 104 \\
\hline
\end{tabular}

Three different affinity cutoff values and two molecular descriptors were used in the assay data curation and classification model training, and only models with MCC $\geq 0.90$ were included in the final $P h-f p$ construction.
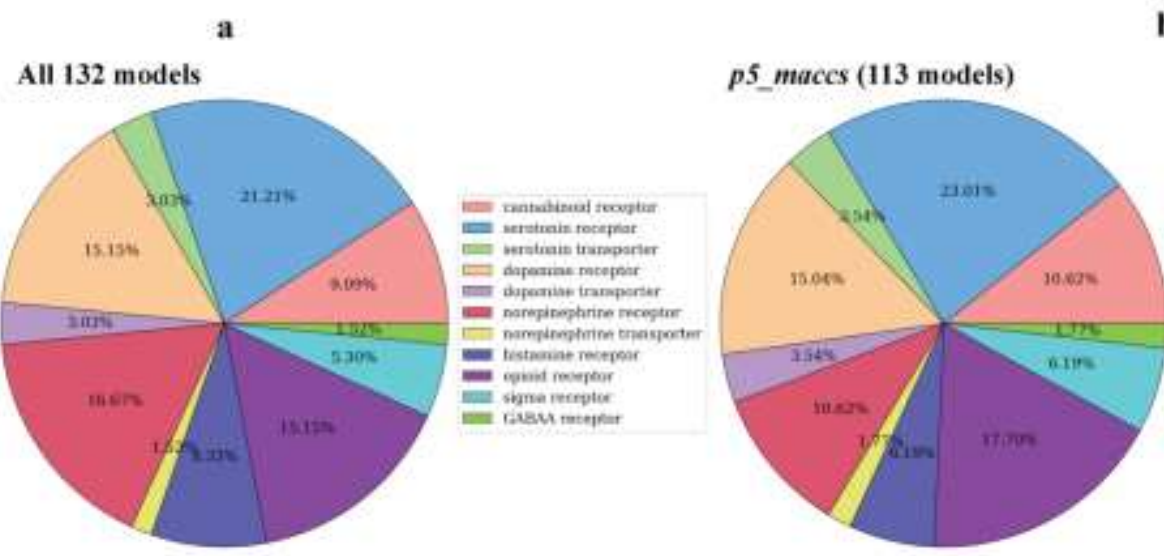

b

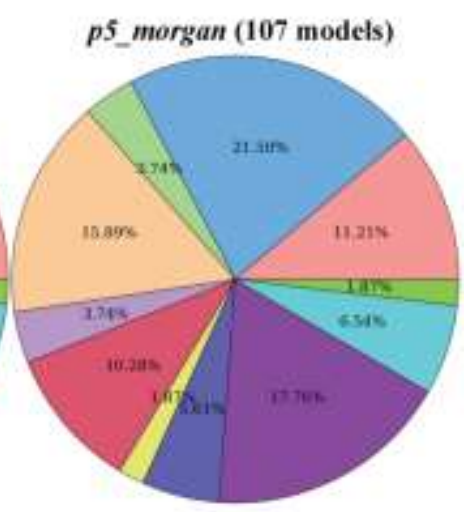

Fig. 2 The representation of 11 molecular target classes for all models and final models selected for the construction of $\mathrm{Ph}-\not p$. a: the total assay datasets trained. b: final models included in constructing p5_maccs and 75 _morgan. The actives in the assay data are defined as compounds with activity values (pKI, pIC50 and pEC50) greater than or equal to 5.

\section{Performance of $P h-f p$ in similarity search}

The distribution of pairwise similarity scores of the NPS set compounds was compared by

calculating Tanimoto coefficients using structural fingerprints MACCS or Morgan, or Rogot-

Goldberg indices using $P h-\not p$ fingerprints, as shown in Fig 3. Fig 3a shows the right-skewed distribution of structural similarity with a medium score of 0.351 and 0.130 using MACCS and Morgan fingerprints, respectively. In contrast, as seen in Fig 3b, the medium pharmacological 
similarity is left-shifted and has an asymmetrical long tail with more pairs of compounds on the high-value side. $25 \%$ of the compound pairs showed pharmacological similarity scores higher than 0.73 and 0.59 using the $p 5 \_$maccs and $p 5$ morgan fingerprints, respectively. In Fig 4, the level of correspondence between structural and pharmacological similarity of the NPS compounds can be demonstrated. The distribution of MACCS and Morgan similarities for all compound pairs, among which the pharmacologically similar pairs are also shown in the bar chart for comparison. In this analysis, pharmacologically similar compound pairs are defined as having a Rogot-Goldberg index greater than or equal to 0.70 using $p^{5} \_$maccs. Only $13.2 \%$ of the $p 5 \_$maccs similar pairs have a MACCS similarity above 0.70 , while $80.4 \%$ of the $\not 5$ maccs similar pairs have a MACCS similarity of between 0.20 and 0.60 . When compared to Morgan, $78.2 \%$ of the $p^{5} \_$maccs similar pairs have a Morgan similarity below 0.30. A similar distribution pattern can be observed when comparing the structural similarity to other $P h-f p$ similarities. 

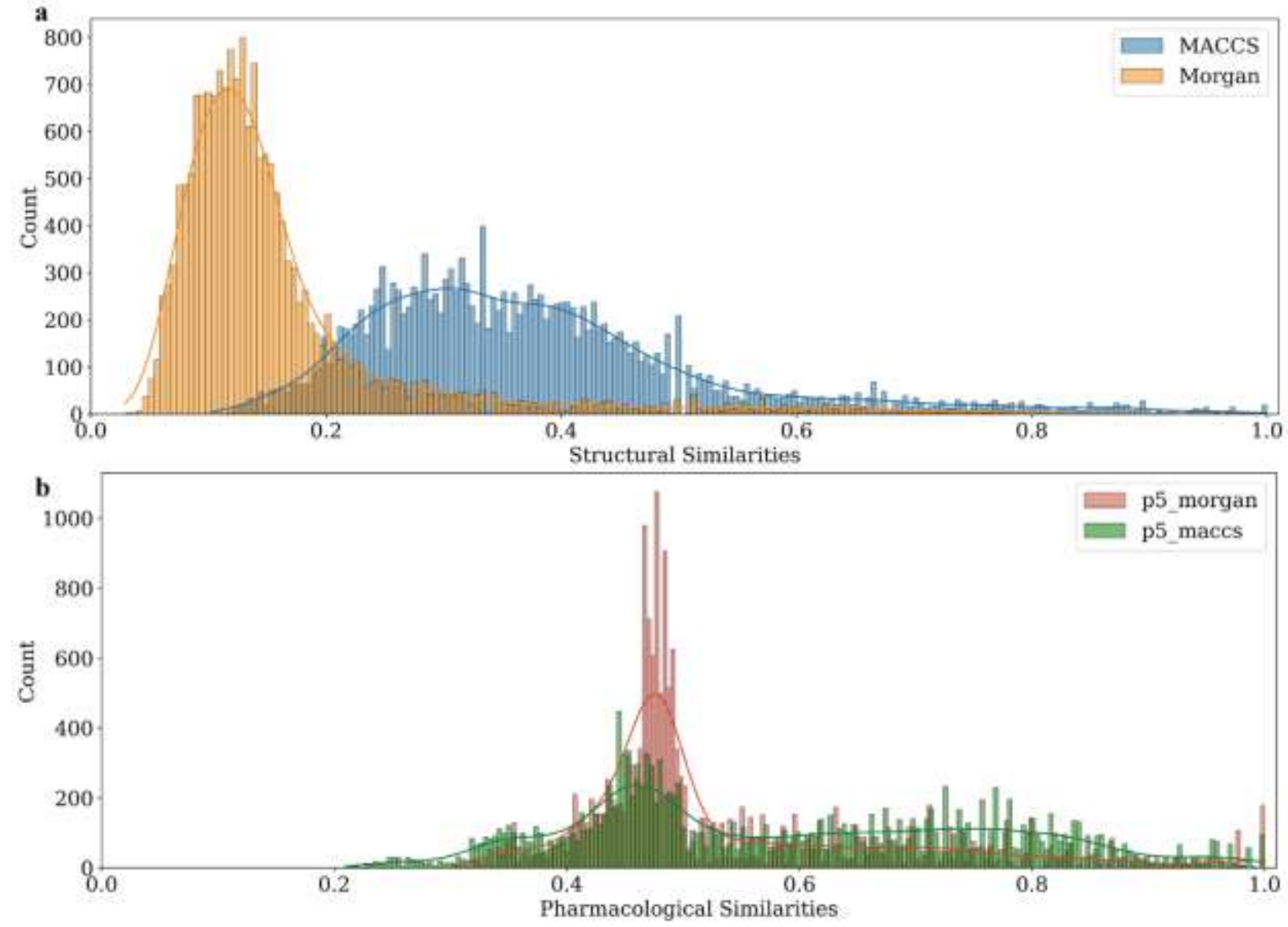

Fig. 3 Frequency distribution of pair-wise comparison of NPS set compounds

a. Structural similarity calculated using Tanimoto coefficient and structural molecular fingerprints, and, b. Pharmacological similarity calculated using Rogot-Goldberg index and pharmacological affinity fingerprints. 


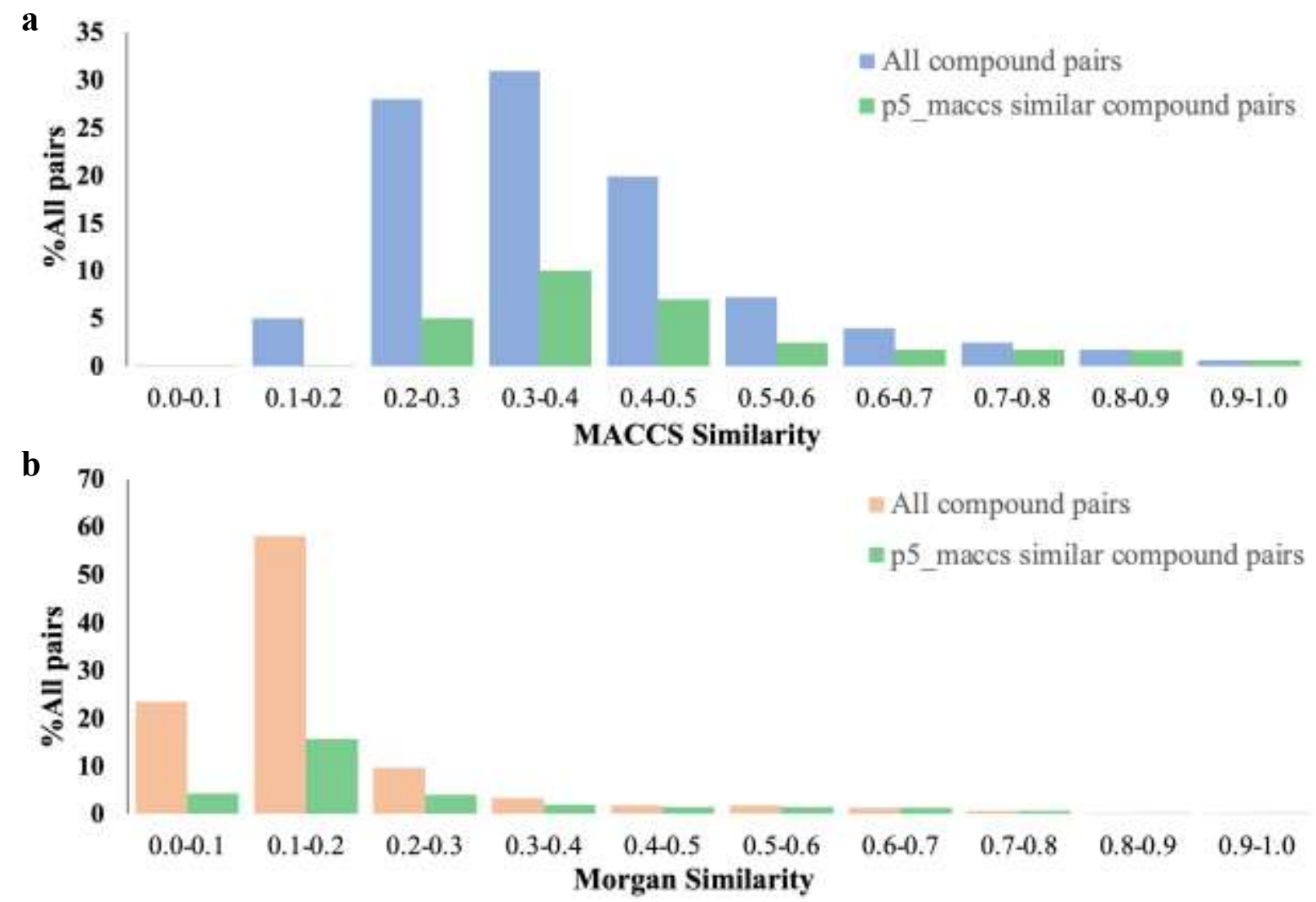

Fig. 4 Distribution of the MACCS, Morgan, and $p 5$ maccs similarity values between $p 5$ maccs similar and $75 \_$maccs unsimilar compound pairs.

In Fig 3b, the pharmacological similarities are centered at 0.45-0.48 for both $p 5$ _morgan and p5_maccs, although $p^{5} \_$morgan regarded more pairs to be median similar, as indicated by the higher peak around this range. Since $p^{5} \_$morgan and 75 maccs fingerprints are similar in length (107 bits vs. 113 bits) and in assay distribution (see Fig 2b), this discrepancy stems from the number of active assays predicted by the classification models using different molecular fingerprints. Naturally, the level of similarity between two compounds is affected by the molecular encoding, as well as the similarity metric used. For instance, in the following example: $A=(00000000)$ and $B=(00000000)$, indicates that both compounds $\mathrm{A}$ and $\mathrm{B}$ are predicted to be inactive in all eight assays. Using the Tanimoto coefficient, their similarity is calculated to be zero. However, the Rogot-Goldberg index is 0.5. Likewise, $\mathrm{A}=(10000000)$ and $\mathrm{B}=(01000000)$ are still considered to be somewhat similar according to the Rogot-Goldberg index of 0.429 since they are both inactive against a total of 6 
assays. In Fig 5, the histogram of the total number of active assay count is plotted for all 189 NPS compounds when described using 25 _maccs and 75 _morgan. Upon further inspection, there are a total of 21 NPS compounds predicted to be inactive in all assays according to $p 5$ morgan, 19 of which are cathinones (stimulants). In general, classification models using Morgan fingerprints as molecular descriptors predict that NPS set compounds are active in fewer assays and result in more "sparse" (few "1s" on the bits) binary $P h-\not p$ fingerprints. Therefore, more compound pairs were calculated as having a Rogot-Goldberg index of about 0.5 using $p^{5} \_$morgan.

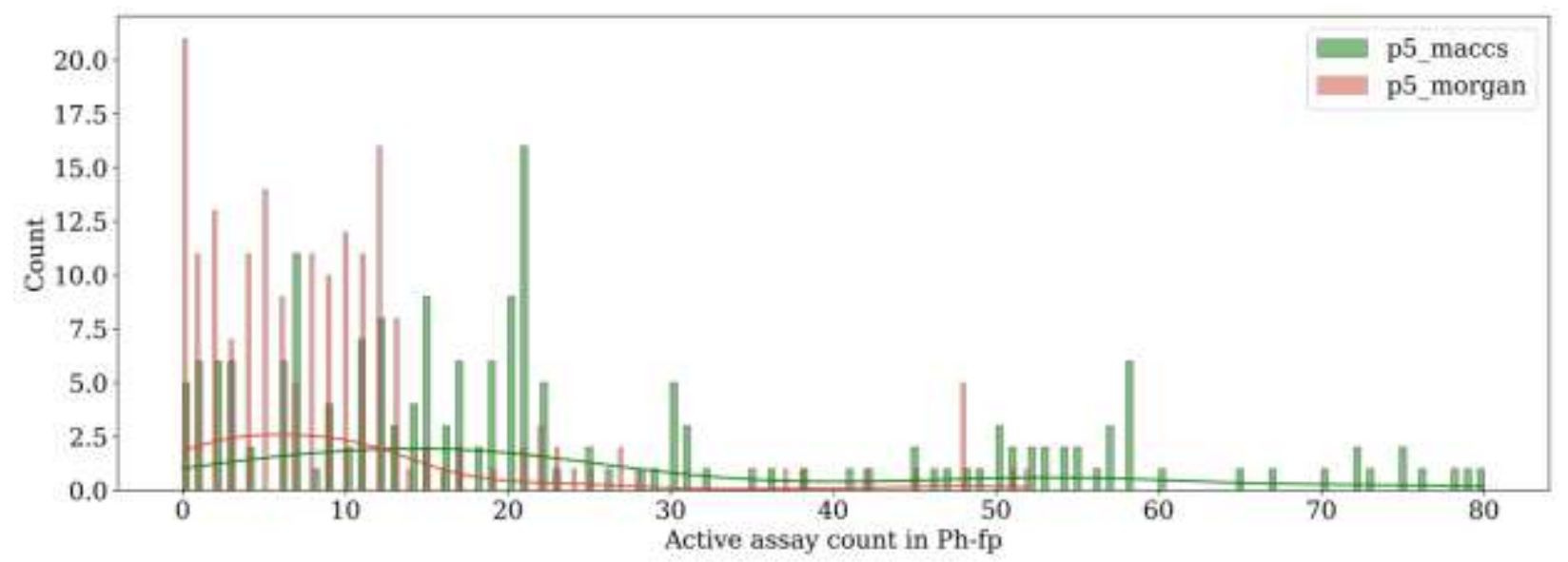

Fig. 5 Histogram of the total number of active assay count (“1” bits) of NPS set compounds

The performance comparison of MACCS and Morgen fingerprints in retrieving compounds of the same pharmacological class is given in Table 4 and is separated for each class. To assess the effect of structural diversity on the similarity search for the retrieval of compounds belonging to the same pharmacological class, a similarity threshold was defined as: $S_{c}=Z \sigma+y$. Here $y$ is the average and $\sigma$ is the standard deviation of the Tanimoto similarity of the $k(=5)$ nearest neighbors of each compound in the pharmacological class. $Z$ is an empirical parameter to control the significant level and set as 0.5 . The smaller the $S_{c}$, the more structurally diverse the compound set. This similarity threshold value was calculated using MACCS and is listed in Table 4 for each pharmacological class. It shows that the compounds considered as stimulants are the most 
structurally diverse, while depressants - benzodiazepines are quite similar to each other. The same conclusion is supported by the $S_{c}$ calculated using Morgan as well. It is then expected that similarity searches using structural fingerprints to identify compounds in the same pharmacological class should perform better when the structural diversity of the compounds is small. This is confirmed by the results. Overall, Morgan had better performance based on EF10 and AUC, but among all pharmacological classes, stimulants were the most difficult to identify by similarity search using structural fingerprints. The optimal threshold was also calculated from the ROC curve for each pharmacological class similarity search, which is defined as the threshold corresponding to the maximal G-Mean $=\sqrt{\operatorname{tp}(1-\mathrm{fp})}$. The lowest similarity thresholds are required to correctly distinguish stimulus-like compounds compared to other classes.

Table 4 Performance comparison of MACCS and Morgan fingerprints in pharmacological class similarity search

\begin{tabular}{|l|c|c|c|c|c|c|c|}
\hline & \multicolumn{4}{|c|}{ MACCS } & \multicolumn{3}{c|}{ Morgan } \\
\hline & EF10 & AUC & Opt_thr & $S_{c}$ & EF10 & AUC & Opt_thr \\
\hline Stimulants & $4.15 \pm 0.81$ & $0.67 \pm 0.07$ & $0.72 \pm 0.03$ & 0.26 & $7.70 \pm 0.78$ & $0.95 \pm 0.03$ & $0.34 \pm 0.02$ \\
\hline Cannabinoids & $7.61 \pm 0.81$ & $0.95 \pm 0.03$ & $0.76 \pm 0.02$ & 0.30 & $8.61 \pm 0.98$ & $0.97 \pm 0.07$ & $0.39 \pm 0.05$ \\
\hline Serotonergic psychedelics & $7.45 \pm 0.86$ & $0.91 \pm 0.06$ & $0.78 \pm 0.03$ & 0.34 & $8.96 \pm 0.70$ & $0.99 \pm 0.03$ & $0.39 \pm 0.05$ \\
\hline D-opioids & $8.23 \pm 1.00$ & $0.98 \pm 0.02$ & $0.77 \pm 0.03$ & 0.43 & $9.26 \pm 0.76$ & $0.99 \pm 0.01$ & $0.44 \pm 0.09$ \\
\hline D-benzodiazepines & $7.55 \pm 2.13$ & $0.94 \pm 0.06$ & $0.80 \pm 0.06$ & 0.52 & $10.1 \pm 1.61$ & $0.99 \pm 0.01$ & $0.50 \pm 0.06$ \\
\hline Average & 7.00 & 0.89 & & & 8.92 & 0.98 & \\
\hline
\end{tabular}

The data shown is the average of 50 similarity searches for each pharmacological class. Both the query and test sets are composed of 1:10 active to decoy ratio by random sampling. Opt_thr is the optimal threshold defined by the maximal G-Mean $=\sqrt{\text { Sensitivity } \times \text { Specificity }}$ 
Table 5 Performance comparison of $P h-f p$ in pharmacological category similarity search

\begin{tabular}{|c|c|c|c|c|c|c|c|c|c|c|c|c|}
\hline & \multirow[b]{2}{*}{ fingerprint } & \multicolumn{5}{|c|}{ MACCS } & \multicolumn{5}{|c|}{ Morgan } & \multirow[b]{2}{*}{ Ave } \\
\hline & & Stimu & Canna & S-psyche & D-opioids & D-benzo & Stimu & Canna & S-psyche & D-opioids & D-benzo & \\
\hline \multirow[t]{6}{*}{ AUC } & p5_maccs & $46.3 \%$ & $0.0 \%$ & $6.6 \%$ & $-1.0 \%$ & $-9.6 \%$ & $3.2 \%$ & $-2.1 \%$ & $-2.0 \%$ & $-2.0 \%$ & $-14.1 \%$ & 0.94 \\
\hline & p6_maccs & $44.8 \%$ & $0.0 \%$ & $4.4 \%$ & $-1.0 \%$ & $-1.1 \%$ & $2.1 \%$ & $-2.1 \%$ & $-4.0 \%$ & $-2.0 \%$ & $-6.1 \%$ & 0.95 \\
\hline & p7_maces & $28.4 \%$ & $0.0 \%$ & $6.6 \%$ & $-1.0 \%$ & $1.1 \%$ & $-9.5 \%$ & $-2.1 \%$ & $-2.0 \%$ & $-2.0 \%$ & $-4.0 \%$ & 0.94 \\
\hline & p5_morgan & $17.9 \%$ & $-3.2 \%$ & $0.0 \%$ & $-6.1 \%$ & $-2.1 \%$ & $-16.8 \%$ & $-5.2 \%$ & $-8.1 \%$ & $-7.1 \%$ & $-7.1 \%$ & 0.89 \\
\hline & p6_morgan & $22.4 \%$ & $2.1 \%$ & $2.2 \%$ & $-4.1 \%$ & $-17.0 \%$ & $-13.7 \%$ & $0.0 \%$ & $-6.1 \%$ & $-5.1 \%$ & $-21.2 \%$ & 0.89 \\
\hline & p7_morgan & $1.5 \%$ & $-2.1 \%$ & $-1.1 \%$ & $-7.1 \%$ & $-55.3 \%$ & $-28.4 \%$ & $-4.1 \%$ & $-9.1 \%$ & $-8.1 \%$ & $-57.6 \%$ & 0.77 \\
\hline \multirow[t]{6}{*}{ EF10 } & p5_maccs & $103.4 \%$ & $0.8 \%$ & $12.1 \%$ & $-3.2 \%$ & $-14.6 \%$ & $9.6 \%$ & $-10.9 \%$ & $-6.8 \%$ & $-13.9 \%$ & $-35.8 \%$ & 7.78 \\
\hline & p6_maccs & $96.1 \%$ & $-6.18 \%$ & $6.4 \%$ & $-0.6 \%$ & $-3.8 \%$ & $5.7 \%$ & $-17.1 \%$ & $-11.5 \%$ & $-11.7 \%$ & $-27.8 \%$ & 7.73 \\
\hline & $p 7$ maccs & $46.8 \%$ & $0.7 \%$ & $5.8 \%$ & $0.7 \%$ & $14.6 \%$ & $-20.9 \%$ & $-11.0 \%$ & $-12.1 \%$ & $-10.5 \%$ & $-13.9 \%$ & 7.71 \\
\hline & p5_morgan & $-31.6 \%$ & $-60.3 \%$ & $-57.6 \%$ & $-57.0 \%$ & $-1.9 \%$ & $-63.1 \%$ & $-64.9 \%$ & $-64.7 \%$ & $-61.8 \%$ & $-26.3 \%$ & 3.99 \\
\hline & p6_morgan & $26.0 \%$ & $1.8 \%$ & $-3.8 \%$ & $-8.0 \%$ & $-21.3 \%$ & $-32.1 \%$ & $-10.0 \%$ & $-20.0 \%$ & $-18.3 \%$ & $-40.9 \%$ & 6.73 \\
\hline & p7_morgan & $-29.9 \%$ & $-45.5 \%$ & $-39.2 \%$ & $-44.7 \%$ & $\underline{-89.3 \%}$ & $-62.2 \%$ & $-51.8 \%$ & $-49.4 \%$ & $-50.9 \%$ & $-91.9 \%$ & 3.39 \\
\hline
\end{tabular}

Stimu $=$ Stimulants, Canna $=$ Cannabinoids, S-psyche $=$ Serotonergic psychedelics, D-opioids $=$ Depressant opioids, D-Benzo $=$

3 Depressant benzodiazepine. The data shown is the average of 50 similarity searches for each pharmacological class using each fingerprint. 
The similarity search results obtained using $P h-f p$ are summarized in Table 5 . The table is

5 divided into two parts, AUC and EF10. For each performance metric, the values in row $i$ and

6 column $j$ of the table represent the percentage difference between the average $P h-f p$ performance

7 minus the average structural fingerprint performance of each pharmacological class. The last column

8 of the table gives the performance of each $P h-f p$ average across all pharmacological classes. Using

9 MACCS or Morgan as a reference, the best results for each performance metric are shown in italics,

10 and the worst results are underlined. In general, no correspondence was observed between the

11 performance of $P h-\not p$ and the structural fingerprints used to construct $P h-f p$. Although Morgan

12 performed best in retrieving NPS compounds of the same pharmacological class, $P h-f p$ constructed

13 with Morgan performed the worst. This can be explained by the lower total active assay counts as

14 demonstrated in Fig 5. During the similarity search, NPS compounds are compared not only with

15 each other but also with decoys. Most decoys were predicted to be inactive in all assays and had all

16 "off" bits in their Ph-fp. The Rogot-Goldberg index between decoys and NPS compounds with

17 sparse "on" bits is still seen as somehow similar. Therefore, it is challenging to efficiently retrieve

18 NPS compounds of the same pharmacological class that also have sparse $\mathrm{Ph}-\mathrm{fp}$. One potential

19 solution is to expand the list of assay datasets used in the construction of $\mathrm{Ph}-\mathrm{fp}$. Another piece of

20 supporting evidence is that $P h-f p$ constructed by using Morgan has the worst performance in

21 identifying the depressant benzodiazepine class compounds. This class of compounds has the lowest

22 degree of structural diversity, however, less than $2 \%$ of the assays in $P h-f p$ are representative of

23 molecular targets specific to this class of compounds.

\section{Performance of Ph-fp in Hierarchical and Spectral Clustering}

25 To calculate the external clustering validation indices ARI and NMI, the externally provided

26 class labels must be used. To reflect the two commonly used categorization systems of NPS

27 compounds, the NPS set is labeled using two sets of external class labels, 1) the five pharmacological 
classes (Stimulants, Cannabinoids, S-psychedelics, D-opioids, and D-benzodiazepines), and 2) the

29 chemical scaffold classes using Maximum Common Substructure (MCS) based approach. The MCS

30 similarity is calculated by identifying structural overlap by matching atomic elements and bond types

31 using the $r d F M C S$ modules implemented in RDKit. ${ }^{[84]}$ The MCS-based clustering was achieved using

32 hierarchical clustering with Ward linkage. A total of 17 classes were determined as the optimal

33 number of clusters by choosing the maximal Silhouette score as the internal validation of the

34 clustering analysis. See Supporting document for more detail. The MCS clustering heatmap in Fig S1

35 shows two supergroups. Under the first supergroup, all depressants - benzodiazepines are in cluster

36 1, where depressants - opioids compounds are split into two clusters (clusters 2 and 9) due to the

37 two main core scaffolds of alkaloid and phenylpiperidine opioids. All cannabinoids are also under

38 this supercluster, with THC based derivatives as one tight cluster and other types of cannabinoids

39 split into several clusters due to the shared indoles, and indazole scaffolds. All serotonergic

40 psychedelic compounds are under the other supercluster and split according to common scaffolds of

41 phenethylamines and tryptamine. Stimulant compounds are distributed in both superclusters due to

42 their structural diversity.

43 For clarity, $K=$ Pharm and $K=$ MCS are used in the following text to refer to the two

44 different external class labels assigned to the NPS set. The fully annotated NPS dataset is available

45 on GitHub repository. Assuming that if $\mathrm{Ph}-\mathrm{fp}$ is indeed describing intrinsic clusters based on the

46 pharmacological characteristics of different classes of NPS compounds, the clustering results using

$47 \quad \mathrm{Ph}-\not p$ fingerprints should be better or at least comparable to structural molecular fingerprints when

48 evaluated using $K=$ Pharm class labels, but worse than structural molecular fingerprints when

49 evaluated using $K=$ MCS class labels.

50 The hyperparameters of both clustering algorithms listed in Table 2 were investigated. In Fig

516 , different linkage methods for hierarchical clustering were used and the validation indices 
52 calculated using external $K=$ MCS and $K=$ Pharm class labels are plotted side-by-side for

53 comparison. Fig 6 is divided into three parts, each corresponding to a clustering validation index.

54 The results show that the performance of hierarchical clustering using the single linkage is poor

55 according to both external and internal validation indices. The single linkage method merges clusters

56 by using the minimum distance between two objects (nearest neighbor) in two clusters and tends to

57 produce the most homogeneous (similar) clusters. As expected, MACCS and Morgan performed

58 significantly better than $\mathrm{Ph}-\mathrm{p}$ in MCS-based cluster discriminations $(K=\mathrm{MCS})$ according to ARI and

59 NMI, with Morgan slightly outperforming MACCS. Most interesting, however, was how their

60 performance changed compared to $P h-f p$ when the task was to distinguish clusters based on

61 pharmacological characteristics $(K=$ Pharm). In the right-hand panels of Fig 6 , it can be seen that

62 although Morgan gives slightly lower ARI and NMI in this task, the scores still indicate moderate

63 accuracy of the results, while MACCS shows worse performance. Also, the performance deviation

64 of $\mathrm{Ph}-\mathrm{fp}$ depends on how it is generated. Therefore, it is recommended to use Morgan as a molecular

65 descriptor to train the classification models to be used for the construction of $\mathrm{Ph}-\mathrm{f}$. Curiously,

66 Morgan had the lowest Silhouette score despite its superior performance in both clustering tasks

67 according to external indices. In contrast, $P h-\not p$ clusterings had the highest Silhouette scores,

68 indicating that, on average, the distance between clusters was the largest and the distance within

69 clusters was the smallest when compounds are described by their pharmacological affinity profiles. 

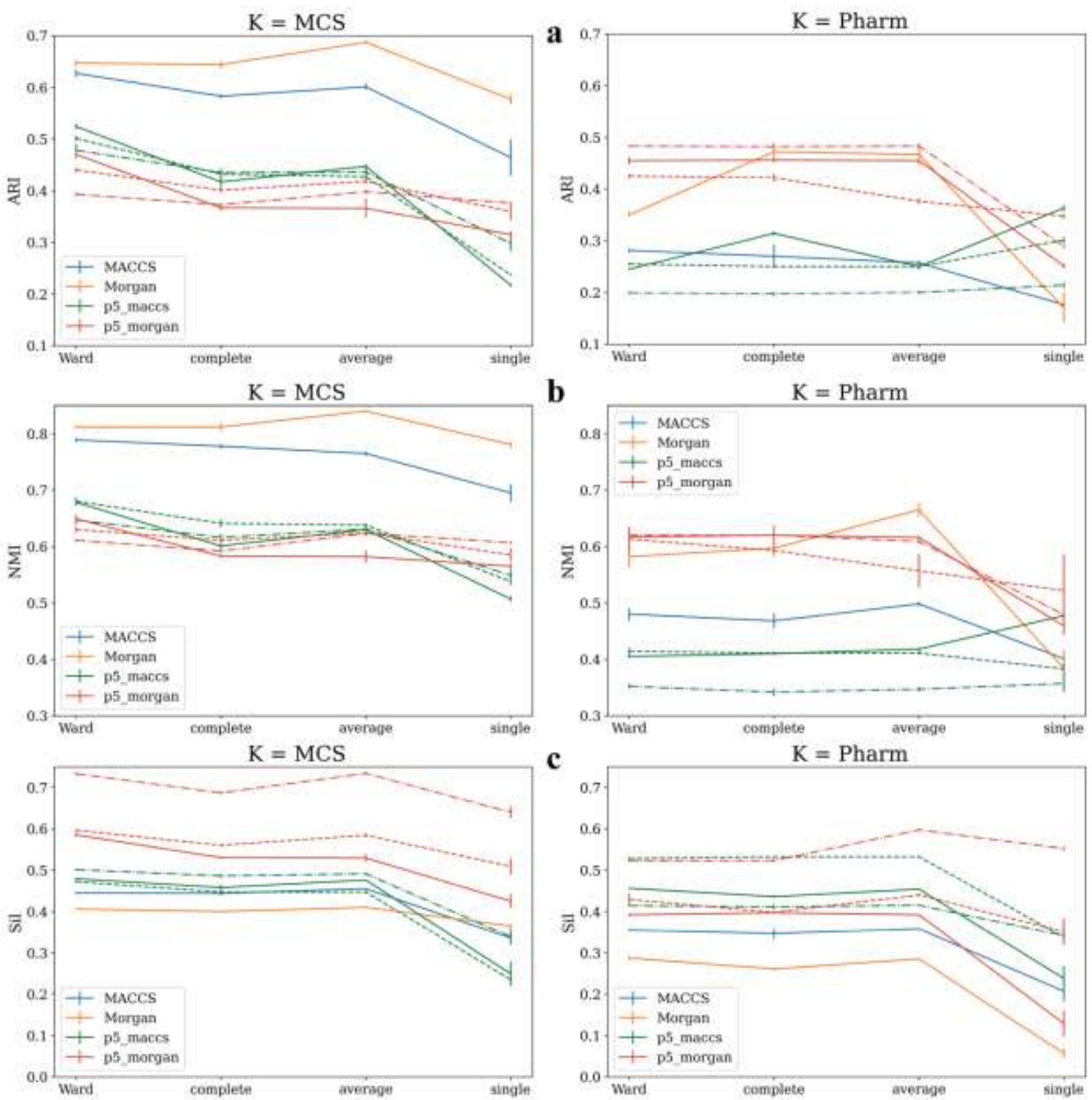

71 Fig. 6 Performance of the hierarchical clustering using different linkage methods.

$72 \mathrm{ARI}$ and NMI are calculated by requesting 5 and 17 clusters and comparing them with external $K=$

73 Pharm and $K=$ MCS class labels. The dashed lines are $p 6 \_m a c c s, p 7 \_m a c c s$ (green) and $p 6 \_m o r g a n$,

74 p7_morgan (red), respectively

In spectral clustering, a grid-search was conducted using two types of similarity graphs $(k$ nearest neighbor graph and fully connected graph), which were evaluated using Silhouette scores.

79 Spectral clustering can be quite sensitive to changes in the similarity graph and the choice of its

80 parameter. In general, if the similarity graph contains more connected components than the number 
81 of clusters we ask the algorithm to detect, then spectral clustering will trivially return connected

82 components as clusters. In the following analysis, the fully connected graph (affinity $=\mathrm{RBF})$ was

83 used. The performance comparison of the two clustering tasks is also shown side-by-side in Fig 7.

84 When constructing the fully connected similarity graph, the Gaussian similarity function is scaled by

85 the parameter gamma (in Scikit-learn implementation), which affects the number of corresponding $k$ -

86 nearest neighbor for each data point. As Luxburg points out, ${ }^{[54]}$ for most data points, the set of

87 neighbors with a similarity significantly greater than 0 should be "not too small and not too large". It

88 can be seen that structural molecular fingerprints, especially Morgan, are sensitive to the parameters

89 used in the construction of similarity graphs, and that their clustering performance deteriorates

90 rapidly when the gamma is increased. While $P h-f p$ is mostly unaffected by the choice of parameters in

91 spectral clustering. However, when using the default parameter gamma $=1$, the same interesting

92 pattern of how the performance "switched" between MACCS and Ph-fp_morgan when the task

93 changed from $K=$ MCS-based clustering and $K=$ Pharm-based clustering. $p 5$ morgan still gives

94 satisfactory performance comparable to Morgan, and overall, $p 5$ morgan has the highest Silhouette

95 scores.

96 

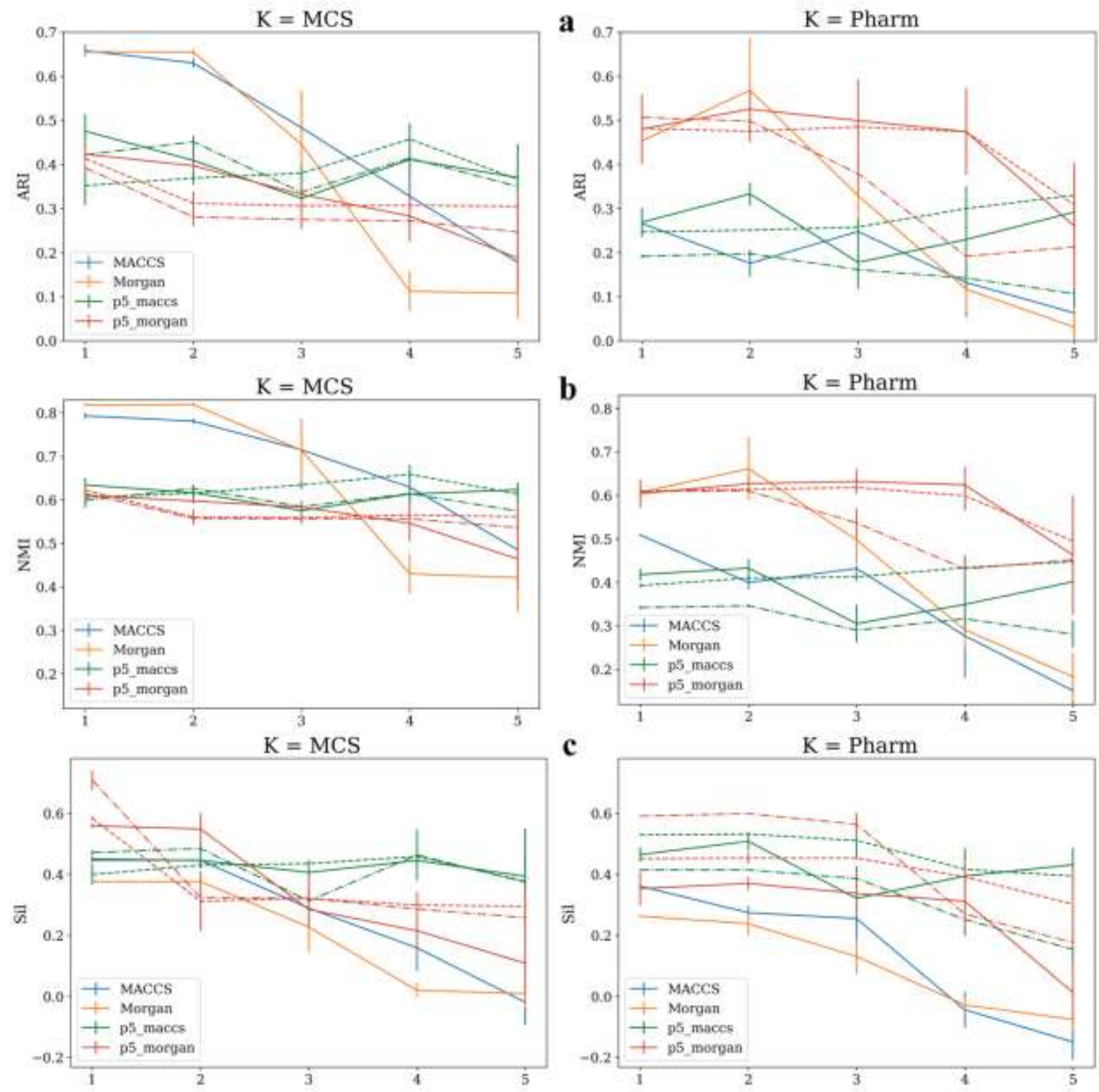

Fig. 7 Performance of the spectral clustering by varying gamma parameter.

99 ARI and NMI are calculated by requesting 5 and 17 clusters and comparing them with external $K=$

100 Pharm and $K=$ MCS class labels. The dashed lines are $p 6 \_$maccs, $p 7$ maccs (green) and $p 6 \_m o r g a n$,

101 p7_morgan (red), respectively

102

103

104

Finally, the performance of the clustering algorithm was verified and compared by setting

105 different values of the requested number of classes $K$. Since the goal is to compare whether the data-

106 driven derived $\mathrm{Ph}-\not \mathrm{p}$ provides a comparable or more optimized clustering performance when the

107 objective is to separate NPS compounds based on their pharmacological characteristics, the external 
108 class label $K=$ Pharm was used to calculate the ARI and NMI external performance indices. The

109 default parameter gamma=1 was used for spectral clustering, and the Ward linkage method was used

110 for hierarchical clustering. The results obtained using hierarchical and spectral clustering algorithms

111 are plotted side-by-side in two panels in Fig 8. The red line indicates the five pharmacological classes

112 of NPS compounds expected from this data. Reasonable results can be expected if the clustering

113 algorithm used is appropriate and the description of the compound is appropriate for the clustering

114 task. The results obtained for both algorithms are mostly similar and in agreement with each other

115 for all fingerprints tested. Using all three validation indices, both Morgan and $p 5$ morgan fingerprints

116 show satisfactory clustering performance, with $p^{5} \_$morgan also showing the highest overall Silhouette

117 score. The highest ARI and NMI were obtained using $p^{5} \_$morgan corresponding to $K=5$.

118 Conversely, setting $\mathrm{K}<10$ resulted in worse performance for hierarchical clustering using MACCS

119 and Morgan. This result suggests that $\mathrm{Ph}-\not p$ constructed using models trained with Morgan

120 fingerprints as input features is best suited to characterize the pharmacological profile of NPS

121 compounds. 

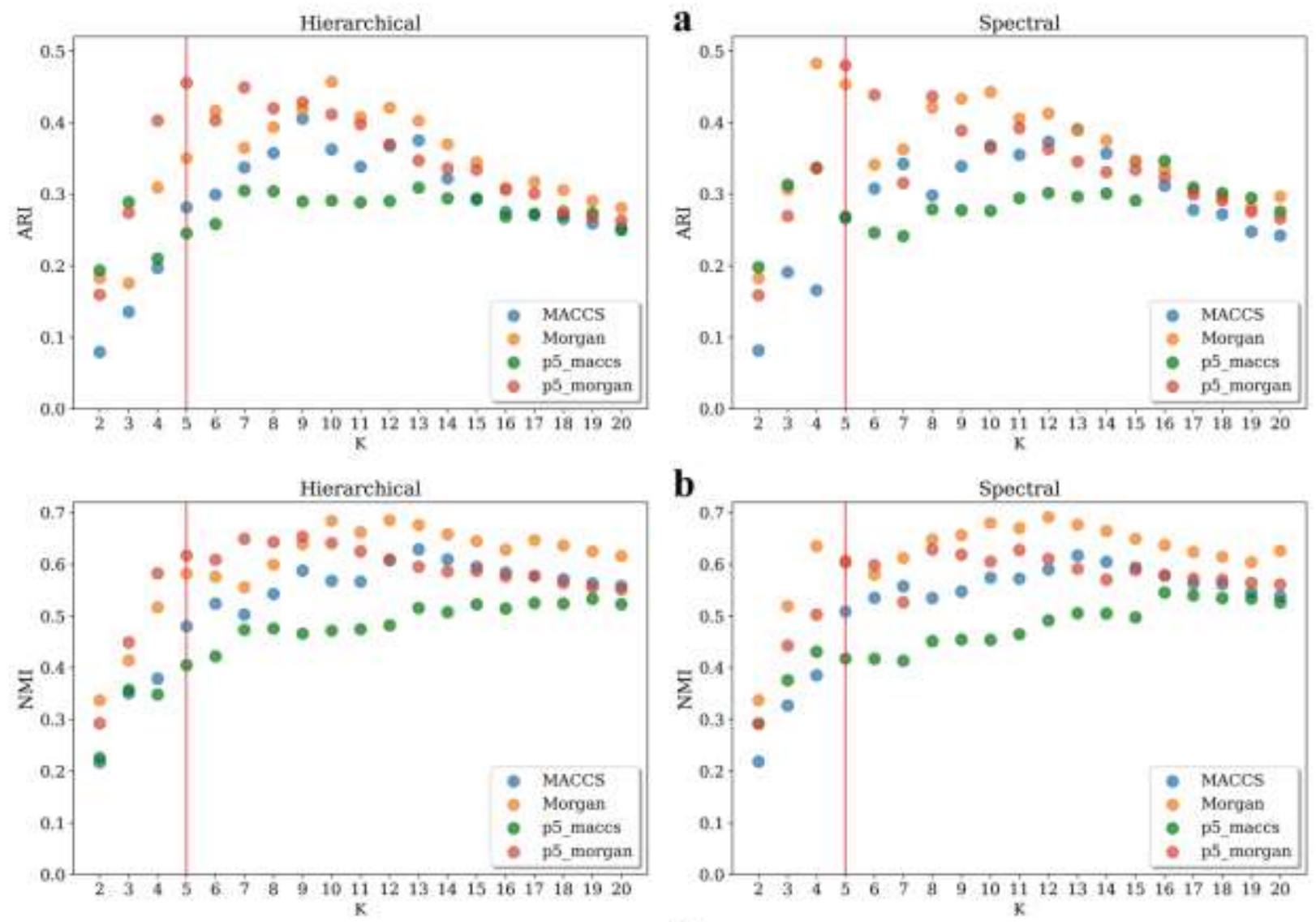

b
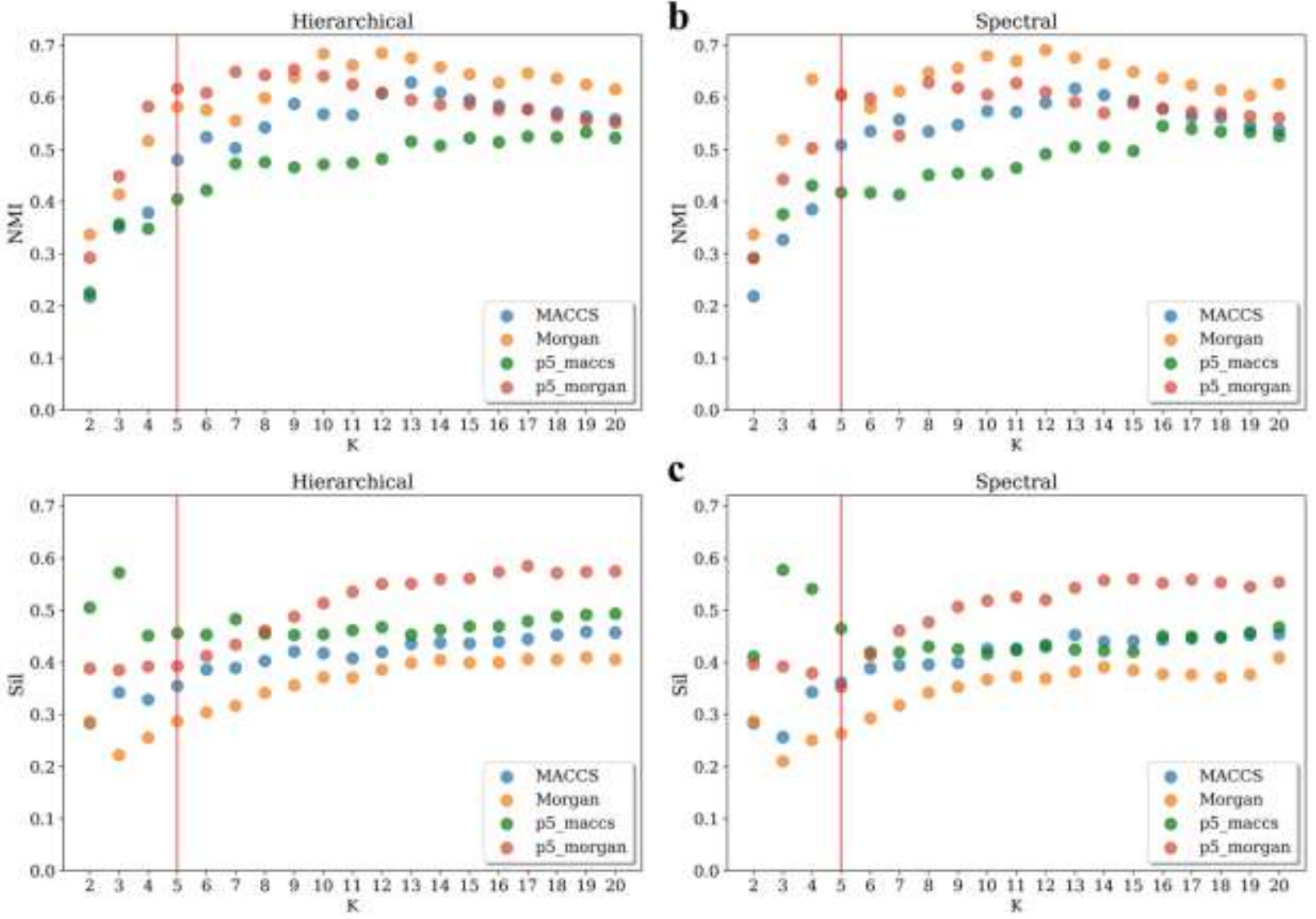

c

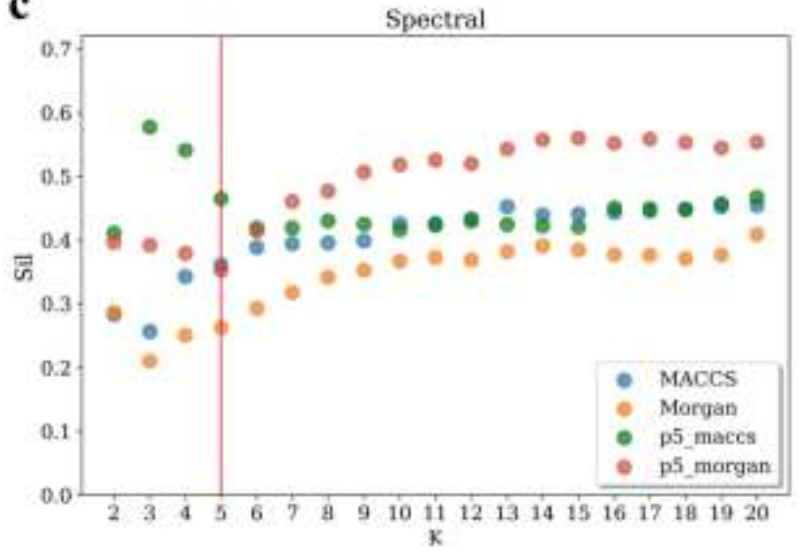

Fig. 8 Performance of the algorithms when varying the expected number of clusters $\mathrm{K}$.

The ARI, NMI, and Silhouette were calculated by comparing to $K=$ Pharm external labels. The red line indicates the five-categories of NPS compounds. The default parameter gamma=1 was used for spectral clustering, and the Ward linkage method was used for hierarchical clustering. 


\section{Conclusion}

The previously unseen NPS continue to emerge at an alarming rate posing additional

135 challenges to their accurate and rapid detection. Given the rapid growth in the number of newly

136 synthesized NPS, it is impractical to study all of them in detail. A more economical approach to

137 mitigate the public health threat of NPS is to rapidly screen for active compounds against molecular

138 targets reported to be responsible for the pharmacological effects of NPS. With the increasing

139 availability of HTS data, predictive models can be constructed for each target individually and then

140 subsequently be used to predict the multi-target pharmacological profile of sample compounds. In

141 this study, a data-driven pharmacological affinity fingerprint $(P h-f p)$ was constructed using ChEMBL

142 bioactivity data with Random Forest classification models. The Ph-fp consists of biological activities

143 predicted across 132 assay datasets. Two different structural molecular fingerprints, MACCS and

144 Morgan, were used as the input feature in the classification models and assay datasets were further

145 curated using different activity threshold values. The performance of $P h-f p$ in similarity searching and 146 unsupervised clustering was evaluated using a set of NPS compounds. The external class labels for

147 NPS set were assigned based on their pharmacological categorization and chemical scaffold

148 categorization. In both tasks, the Ph-fp was compared to 1024 bits long Morgan and 116 bits on

149 MACCS structural molecular fingerprints.

The degree of similarity between pairs of compounds is strongly influenced by the encoding

151 of molecular fingerprints, and the use of $\mathrm{Ph}-\mathrm{p}$ to encode compounds' predicted pharmacological

152 affinity profiles can provide a complementary perspective when screening for compounds that have

153 the potential to become the next emerging NPS. Ph-fp outperformed MACCS in the similarity search

154 in retrieving stimulants with the highest level of compound structural diversity. The poor

155 performance of the $P h-\not p$ constructed by the model using Morgan fingerprints as input features

156 demonstrates the importance of expanding the list of assays. Using the Rogot-Goldberg index as the 
similarity metric overestimated the level of similarity predicted between compounds with fewer "on"

158 bits in their $P h-f p$ and decoys. However, when clustering only the NPS compound set without

159 decoys, $P h-f p$ trained with Morgan can successfully discriminate compounds based on generally

160 accepted pharmacological categorization, with overall superior performance using both external and

161 internal clustering validation indices. In summary, data-driven $\mathrm{Ph}-\mathrm{FP}$ is a promising tool for

162 screening potential emerging NPS compounds using public domain bioassay data. It would be

163 interesting to compare the performance of representative databases constructed using structure-

164 based clustering alone or in combination with the pharmacological space of the NPS in identifying

165 unknown samples.

\section{Statements and Declarations}

167

168

\section{Funding}

Computational resources were provided in part by the MERCURY consortium

(http://mercuryconsortium.org/) under National Science Foundation grants CHE-1229354, CHE1662030, and CHE-2018427.

\section{Competing interests}

The authors declare no competing financial interest.

\section{Data and Software Availability}

The dataset(s) and python source code supporting the conclusions of this article are available in the GitHub repository, https://github.com/nina23bom/NPS-Pharmacological-profile-fingerprintprediction-using-ML

\section{$180 \quad$ References}

181 1. UNODC Early Warning Advisory on New Psychoactive Substances. What are NPS? 182 https://www.unodc.org/LSS/Home/NPS. (accessed Mar 2021).

183 2. "Title 21 United States Code (USC) Controlled Substances Act" United States Drug 184 Enforcement Administration:

185 https://www.dea.gov/controlled-substances-act. (accessed Mar 2021).

186 3. Schifano, F.; Napoletano, F.; Chiappini, S.; Guirguis, A.; Corkery, J. M.; Bonaccorso, S.; 187 Ricciardi, A.; Scherbaum, N.; Vento, A. (2021) New/emerging psychoactive substances and 188 associated psychopathological consequences. Psychol. Med. 51 (1), 30-42.

189 4. PSA 2016 Psychoactive Substances Act 2016:

190 https://www.legislation.gov.uk/ukpga/2016/2/contents/enacted. (accessed May 2021). 
5. Baldwin, G. T.; Seth, P.; Noonan, R. K. (2021) Continued Increases in Overdose Deaths Related to Synthetic Opioids: Implications for Clinical Practice. JAMA Health Forum 325 (12), 1151-1152.

194 6. Muhamadali, H.; Watt, A.; Xu, Y.; Chisanga, M.; Subaihi, A.; Jones, C.; Ellis, D. I.; 195 Sutcliffe, O. B.; Goodacre, R. (2019) Rapid Detection and Quantification of Novel Psychoactive Substances (NPS) Using Raman Spectroscopy and Surface-Enhanced Raman Scattering. Front. Chem. 7 (412).

198 7. Shafi, A.; Berry, A. J.; Sumnall, H.; Wood, D. M.; Tracy, D. K. (2020) New psychoactive substances: a review and updates. Ther. Adv. Psychopharmacol. 10, 20451253209671972045125320967197.

201 8. Tracy, D. K.; Wood, D. M.; Baumeister, D. (2017) Novel psychoactive substances: types, 202 mechanisms of action, and effects. BMJ 356, i6848.

203 9. Miliano, C.; Serpelloni, G.; Rimondo, C.; Mereu, M.; Marti, M.; De Luca, M. A. (2016) Neuropharmacology of New Psychoactive Substances (NPS): Focus on the Rewarding and Reinforcing Properties of Cannabimimetics and Amphetamine-Like Stimulants. Front Neurosci 10, 153-153. 10. European Monitoring Centre for Drugs and Drug Addiction. European drug report 2019: trends and developments, http://www.emcdda.europa.eu/system/files/publications/11364/20191724_TDAT19001ENN_P DF.pdf (2019). (accessed Dec 2021).

11. Coppola, M.; Mondola, R. (2012) Synthetic cathinones: chemistry, pharmacology and toxicology of a new class of designer drugs of abuse marketed as "bath salts" or "plant food". Toxicol. Lett. 211 (2), 144-9.

12. Luethi, D.; Liechti, M. E. (2020) Designer drugs: mechanism of action and adverse effects. Arch. Toxicol. 94 (4), 1085-1133. of plasma membrane monoamine transporters by beta-ketoamphetamines. Eur. J. Pharmacol.

219 14. Marusich, J. A.; Antonazzo, K. R.; Wiley, J. L.; Blough, B. E.; Partilla, J. S.; Baumann, M. H. (2014) Pharmacology of novel synthetic stimulants structurally related to the "bath salts" constituent 3,4-methylenedioxypyrovalerone (MDPV). Neuropharmacology 87, 206-13. 15. Baumann, M. H.; Ayestas, M. A., Jr.; Partilla, J. S.; Sink, J. R.; Shulgin, A. T.; Daley, P. F.; Brandt, S. D.; Rothman, R. B.; Ruoho, A. E.; Cozzi, N. V. (2012) The designer methcathinone analogs, mephedrone and methylone, are substrates for monoamine transporters in brain tissue. Neuropsychopharmacology 37 (5), 1192-1203.

16. Cameron, K.; Kolanos, R.; Vekariya, R.; De Felice, L.; Glennon, R. A. (2013) Mephedrone and methylenedioxypyrovalerone (MDPV), major constituents of "bath salts," produce opposite effects at the human dopamine transporter. Psychopharmacology (Berl) 227 (3), 493-9. 17. Banister, S. D.; Connor, M., The Chemistry and Pharmacology of Synthetic Cannabinoid Receptor Agonists as New Psychoactive Substances: Origins. In: Maurer H., Brandt S. (eds) New Psychoactive Substances. Handbook of Experimental Pharmacology. Springer: 2018; Vol. 252, pp 165-190.

233 18. Le Boisselier, R.; Alexandre, J.; Lelong-Boulouard, V.; Debruyne, D. (2017) Focus on

234 cannabinoids and synthetic cannabinoids. Clin. Pharmacol. Ther. 101 (2), 220-229. 
19. Finlay, D. B.; Manning, J. J.; Ibsen, M. S.; Macdonald, C. E.; Patel, M.; Javitch, J. A.;

236

237

238

239

240

241

242

243

244

245

246

247

248

249

250

251

252

253

254

255

256

257

258

259

260

261

262

263

264

265

266

267

268

269

270

271

272

273

274

275

276

277

Banister, S. D.; Glass, M. (2019) Do Toxic Synthetic Cannabinoid Receptor Agonists Have Signature in Vitro Activity Profiles? A Case Study of AMB-FUBINACA. ACS Chem. Neurosci. 10 (10), 4350-4360.

20. Silva, J. P.; Araújo, A. M.; de Pinho, P. G.; Carmo, H.; Carvalho, F. (2019) Synthetic Cannabinoids JWH-122 and THJ-2201 Disrupt Endocannabinoid-Regulated Mitochondrial Function and Activate Apoptotic Pathways as a Primary Mechanism of In Vitro Nephrotoxicity at In Vivo Relevant Concentrations. Toxicol. Sci. 169 (2), 422-435.

21. Kolaczynska, K. E.; Luethi, D.; Trachsel, D.; Hoener, M. C.; Liechti, M. E. (2019) Receptor Interaction Profiles of 4-Alkoxy-Substituted 2,5-Dimethoxyphenethylamines and Related Amphetamines. Front. Pharmacol. 10 (1423).

22. Rickli, A.; Luethi, D.; Reinisch, J.; Buchy, D.; Hoener, M. C.; Liechti, M. E. (2015)

Receptor interaction profiles of novel N-2-methoxybenzyl (NBOMe) derivatives of 2,5dimethoxy-substituted phenethylamines (2C drugs). Neuropharmacology 99, 546-53.

23. Nichols, D. E. (2016) Psychedelics. Pharmacol. Rev. 68 (2), 264-355.

24. Eshleman, A. J.; Wolfrum, K. M.; Reed, J. F.; Kim, S. O.; Johnson, R. A.; Janowsky, A. (2018) Neurochemical pharmacology of psychoactive substituted N-benzylphenethylamines: High potency agonists at 5-HT(2A) receptors. Biochem. Pharmacol. (Amsterdam, Neth.) 158, 2734.

25. Tittarelli, R.; Mannocchi, G.; Pantano, F.; Romolo, F. S. (2015) Recreational use, analysis and toxicity of tryptamines. Curr. Neuropharmacol. 13 (1), 26-46.

26. Luethi, D.; Liechti, M. E. (2018) Monoamine Transporter and Receptor Interaction Profiles in Vitro Predict Reported Human Doses of Novel Psychoactive Stimulants and Psychedelics. Int. J. Neuropsychopharmacol. 21 (10), 926-931.

27. Luethi, D.; Trachsel, D.; Hoener, M. C.; Liechti, M. E. (2018) Monoamine receptor interaction profiles of 4-thio-substituted phenethylamines (2C-T drugs). Neuropharmacology 134 (Pt A), 141-148.

28. Rickli, A.; Moning, O. D.; Hoener, M. C.; Liechti, M. E. (2016) Receptor interaction profiles of novel psychoactive tryptamines compared with classic hallucinogens. Eur. Neuropsychopharmacol. 26 (8), 1327-37.

29. Wagmann, L.; Brandt, S. D.; Stratford, A.; Maurer, H. H.; Meyer, M. R. (2019) Interactions of phenethylamine-derived psychoactive substances of the $2 \mathrm{C}$-series with human monoamine oxidases. Drug Test. Anal. 11 (2), 318-324.

30. Blough, B. E.; Landavazo, A.; Decker, A. M.; Partilla, J. S.; Baumann, M. H.; Rothman, R. B. (2014) Interaction of psychoactive tryptamines with biogenic amine transporters and serotonin receptor subtypes. Psychopharmacology 231 (21), 4135-4144.

31. Cozzi, N. V.; Gopalakrishnan, A.; Anderson, L. L.; Feih, J. T.; Shulgin, A. T.; Daley, P. F.; Ruoho, A. E. (2009) Dimethyltryptamine and other hallucinogenic tryptamines exhibit substrate behavior at the serotonin uptake transporter and the vesicle monoamine transporter. J. Neural Transm. 116 (12), 1591-9.

32. Waters, L.; Manchester, K. R.; Maskell, P. D.; Haegeman, C.; Haider, S. (2018) The use of a quantitative structure-activity relationship (QSAR) model to predict GABA-A receptor binding of newly emerging benzodiazepines. Sci. Justice 58 (3), 219-225. 
33. Manchester, K. R.; Lomas, E. C.; Waters, L.; Dempsey, F. C.; Maskell, P. D. (2018) The emergence of new psychoactive substance (NPS) benzodiazepines: A review. Drug Test. Anal. 10 (1), 37-53.

281 34. Bodnar, R. J. (2021) Endogenous opiates and behavior: 2019. Peptides 141, 170547.

282 35. Suzuki, J.; El-Haddad, S. (2017) A review: Fentanyl and non-pharmaceutical fentanyls.

283 Drug Alcohol Depend. 171, 107-116.

284 36. Armenian, P.; Vo, K. T.; Barr-Walker, J.; Lynch, K. L. (2018) Fentanyl, fentanyl analogs and novel synthetic opioids: A comprehensive review. Neuropharmacology 134, 121-132. 37. Baumann, M. H.; Majumdar, S.; Le Rouzic, V.; Hunkele, A.; Uprety, R.; Huang, X. P.; Xu, J.; Roth, B. L.; Pan, Y.-X.; Pasternak, G. W. (2018) Pharmacological characterization of novel 101-107.

\section{CPS 2018 Crown Prosecution Service (2018). https://www.cps.gov.uk/legal-} guidance/psychoactive-substances. (accessed May 2021).

39. McInnes, C. (2007) Virtual screening strategies in drug discovery. Curr. Opin. Chem. Biol. 11 (5), 494-502.

40. Chen, B.; Harrison, R. F.; Papadatos, G.; Willett, P.; Wood, D. J.; Lewell, X. Q.; Greenidge, P.; Stiefl, N. (2007) Evaluation of machine-learning methods for ligand-based virtual screening. J. Comput.-Aided Mol. Des. 21 (1-3), 53-62.

41. Chung, H.; Choi, H.; Heo, S.; Kim, E.; Lee, J. (2013) Synthetic cannabinoids abused in South Korea: drug identifications by the National Forensic Service from 2009 to June 2013. Forensic Toxicol. 32, 82-88.

42. Sobolevsky, T.; Prasolov, I.; Rodchenkov, G. (2012) Detection of urinary metabolites of AM-2201 and UR-144, two novel synthetic cannabinoids. Drug Test. Anal. 4 (10), 745-53. 43. Banister, S. D.; Kevin, R. C.; Martin, L.; Adams, A.; Macdonald, C.; Manning, J. J.; Boyd, R.; Cunningham, M.; Stevens, M. Y.; McGregor, I. S. (2019) The chemistry and pharmacology of putative synthetic cannabinoid receptor agonist (SCRA) new psychoactive substances (NPS) 5F - PY - PICA, 5F - PY - PINACA, and their analogs. Drug Test. Anal. 11 (7), 976-989.

44. Wiley, J. L.; Lefever, T. W.; Marusich, J. A.; Grabenauer, M.; Moore, K. N.; Huffman, J. W.; Thomas, B. F. (2016) Evaluation of first generation synthetic cannabinoids on binding at non-cannabinoid receptors and in a battery of in vivo assays in mice. Neuropharmacology 110 (Pt A), 143-153.

45. Wassermann, A. M.; Lounkine, E.; Davies, J. W.; Glick, M.; Camargo, L. M. (2015) The opportunities of mining historical and collective data in drug discovery. Drug Discovery Today 20 (4), $422-434$.

46. Shoemaker, R. H. (2006) The NCl60 human tumour cell line anticancer drug screen. Nat. Rev. Cancer 6 (10), 813-823.

47. Riniker, S.; Wang, Y.; Jenkins, J. L.; Landrum, G. A. (2014) Using Information from Historical High-Throughput Screens to Predict Active Compounds. J. Chem. Inf. Model. 54 (7), 1880-1891.

48. Wang, Y.; Bryant, S. H.; Cheng, T.; Wang, J.; Gindulyte, A.; Shoemaker, B. A.; Thiessen, P. A.; He, S.; Zhang, J. (2017) PubChem BioAssay: 2017 update. Nucleic Acids Res. 45 (D1), D955-d963. 
49. Helal, K. Y.; Maciejewski, M.; Gregori-Puigjané, E.; Glick, M.; Wassermann, A. M. (2016) Public Domain HTS Fingerprints: Design and Evaluation of Compound Bioactivity Profiles from PubChem's Bioassay Repository. J. Chem. Inf. Model. 56 (2), 390-398. 50. Nepusz, T.; Sasidharan, R.; Paccanaro, A. (2010) SCPS: a fast implementation of a spectral method for detecting protein families on a genome-wide scale. BMC Bioinf. 11 (1), 120. 51. Sgourakis, N. G.; Merced-Serrano, M.; Boutsidis, C.; Drineas, P.; Du, Z.; Wang, C.; Garcia, A. E. (2011) Atomic-level characterization of the ensemble of the $A \beta(1-42)$ monomer in water using unbiased molecular dynamics simulations and spectral algorithms. J. Mol. Biol. 405 (2), 570-83.

52. Yu, Z.; Li, L.; You, J.; Wong, H. S.; Han, G. (2012) SC ST $^{3}$ Triple Spectral Clustering-Based Consensus Clustering Framework for Class Discovery from Cancer Gene Expression Profiles. IEEE/ACM Trans. Comp. Biol. Bioinf. 9 (6), 1751-1765. 53. Brewer, M. L. (2007) Development of a spectral clustering method for the analysis of molecular data sets. J. Chem. Inf. Model. 47 (5), 1727-33. 54. von Luxburg, U. (2007) A tutorial on spectral clustering. Statist. Comput. 17 (4), 395-416. 55. Williams, A. J.; Ekins, S.; Tkachenko, V. (2012) Towards a gold standard: regarding quality in public domain chemistry databases and approaches to improving the situation. Drug Discovery Today 17 (13-14), 685-701. 56. Kramer, C.; Kalliokoski, T.; Gedeck, P.; Vulpetti, A. (2012) The Experimental Uncertainty of Heterogeneous Public Ki Data. J. Med. Chem. 55 (11), 5165-5173. 57. Rickli, A.; Kopf, S.; Hoener, M. C.; Liechti, M. E. (2015) Pharmacological profile of novel psychoactive benzofurans. Br. J. Pharmacol. 172 (13), 3412-3425.

58. Luethi, D.; Kaeser, P. J.; Brandt, S. D.; Krähenbühl, S.; Hoener, M. C.; Liechti, M. E. (2018) Pharmacological profile of methylphenidate-based designer drugs. Neuropharmacology 134 (Pt A), 133-140.

59. Luethi, D.; Kolaczynska, K. E.; Docci, L.; Krähenbühl, S.; Hoener, M. C.; Liechti, M. E. (2018) Pharmacological profile of mephedrone analogs and related new psychoactive substances. Neuropharmacology 134, 4-12.

60. Simmler, L. D.; Rickli, A.; Hoener, M. C.; Liechti, M. E. (2014) Monoamine transporter and receptor interaction profiles of a new series of designer cathinones. Neuropharmacology 79, 152-60.

61. Mysinger, M. M.; Carchia, M.; Irwin, J. J.; Shoichet, B. K. (2012) Directory of Useful Decoys, Enhanced (DUD-E): Better Ligands and Decoys for Better Benchmarking. J. Med. Chem. 55 (14), 6582-6594.

62. Irwin, J. J.; Shoichet, B. K. (2005) ZINC - A Free Database of Commercially Available Compounds for Virtual Screening. J. Chem. Inf. Model. 45 (1), 177-182.

360 64. Yu, P.; Wild, D. J. (2012) Fast rule-based bioactivity prediction using associative 361 classification mining. J. Cheminf. 4 (1), 29-29.

362 65. Rogers, D.; Hahn, M. (2010) Extended-Connectivity Fingerprints. J. Chem. Inf. Model. 50 363 (5), 742-754. 
66. Hert, J.; Willett, P.; Wilton, D. J.; Acklin, P.; Azzaoui, K.; Jacoby, E.; Schuffenhauer, A. (2004) Comparison of topological descriptors for similarity-based virtual screening using multiple bioactive reference structures. Org. Biomol. Chem. 2 (22), 3256-66. 67. Gardiner, E. J.; Holliday, J. D.; O'Dowd, C.; Willett, P. (2011) Effectiveness of 2D fingerprints for scaffold hopping. Future Med. Chem. 3 (4), 405-14. 68. Varin, T.; Bureau, R.; Mueller, C.; Willett, P. (2009) Clustering files of chemical structures using the Székely-Rizzo generalization of Ward's method. J. Mol. Graphics Modell. 28 (2), 187-195.

69. Breiman, L. (2001) Random Forests. Mach. Learn. 45 (1), 5-32.

70. Svetnik, V.; Liaw, A.; Tong, C.; Culberson, J. C.; Sheridan, R. P.; Feuston, B. P. (2003) Random Forest: A Classification and Regression Tool for Compound Classification and QSAR Modeling. J. Chem. Inf. Comput. Sci. 43 (6), 1947-1958.

71. Varma, S.; Simon, R. (2006) Bias in error estimation when using cross-validation for model selection. BMC Bioinf. 7 (1), 91.

72. Baldi, P.; Brunak, S.; Chauvin, Y.; Andersen, C. A. F.; Nielsen, H. (2000) Assessing the accuracy of prediction algorithms for classification: an overview. Bioinformatics 16 (5), 412-424. (MCC) over F1 score and accuracy in binary classification evaluation. BMC Genomics 21 (1), 6. 74. Simmler, L. D.; Buser, T. A.; Donzelli, M.; Schramm, Y.; Dieu, L. H.; Huwyler, J.; Chaboz, S.; Hoener, M. C.; Liechti, M. E. (2013) Pharmacological characterization of designer cathinones in vitro. Br. J. Pharmacol. 168 (2), 458-70.

\section{Simmler, L. D.; Rickli, A.; Schramm, Y.; Hoener, M. C.; Liechti, M. E. (2014)}

Pharmacological profiles of aminoindanes, piperazines, and pipradrol derivatives. Biochem. Pharmacol. (Amsterdam, Neth.) 88 (2), 237-44.

76. Rickli, A.; Hoener, M. C.; Liechti, M. E. (2015) Monoamine transporter and receptor interaction profiles of novel psychoactive substances: para-halogenated amphetamines and pyrovalerone cathinones. Eur. Neuropsychopharmacol. 25 (3), 365-76.

77. Luethi, D.; Hoener, M. C.; Liechti, M. E. (2018) Effects of the new psychoactive substances diclofensine, diphenidine, and methoxphenidine on monoaminergic systems. Eur J Pharmacol 819, 242-247.

78. Rogot, E.; Goldberg, I. D. (1966) A proposed index for measuring agreement in testretest studies. J Chronic Dis 19 (9), 991-1006.

79. Bajusz, D.; Rácz, A.; Héberger, K. (2015) Why is Tanimoto index an appropriate choice for fingerprint-based similarity calculations? J. Cheminf. 7 (1), 20.

80. Rousseeuw, P. J. (1987) Silhouettes: A graphical aid to the interpretation and validation of cluster analysis. J. Comput. Appl. Math. 20, 53-65.

81. Lei, Y.; Bezdek, J. C.; Romano, S.; Vinh, N. X.; Chan, J.; Bailey, J. (2017) Ground truth bias in external cluster validity indices. Pattern Recognition 65, 58-70.

82. Hubert, L.; Arabie, P. (1985) Comparing partitions. J. Classif. 2 (1), 193-218.

83. Strehl, A.; Ghosh, J. (2002) Cluster Ensembles --- A Knowledge Reuse Framework for Combining Multiple Partitions. J. Mach. Learn. Res. 3, 583-617.

84. Zhang, B.; Vogt, M.; Maggiora, G. M.; Bajorath, J. (2015) Design of chemical space networks using a Tanimoto similarity variant based upon maximum common substructures. $J$. Comput.-Aided Mol. Des. 29 (10), 937-950. 
408

409 


\section{Supplementary Files}

This is a list of supplementary files associated with this preprint. Click to download.

- SupportingMaterialJan2022.docx 